\title{
ON THE STRUCTURE OF BANACH ALGEBRAS ASSOCIATED WITH AUTOMORPHISMS
}

\author{
A. V. LEBEDEV
}

\begin{abstract}
We study the structure of the Banach algebra $B\left(A, T_{g}\right)$ generated by a Banach algebra $A$ of operators in a Banach space $D$ and a group $\left\{T_{g}\right\}_{g \in G}$ of isometries of $D$ such that $T_{g} A T_{g}^{-1}=A$. We analyze relations between the existence of an expectation from $B\left(A, T_{g}\right)$ to $A$, topological (metric) freeness of the action by automorphisms of the algebra $A$ induced by $T_{g}$, and the dual action of the group $G$ on $B\left(A, T_{g}\right)$. The obtained results are applied to the description of the structure of Banach algebras generated by "weighted composition operators" in various spaces.
\end{abstract}

\section{INTRODUCTION}

The main object to be studied in this paper is the Banach algebra $B\left(A, T_{g}\right)$ generated by a Banach algebra $A$ of operators acting in a Banach space $D$ and the group $\left\{T_{g}\right\}_{g \in G}$ of isometries of $D$ (a representation $G \ni g \rightarrow T_{g}$ of a discrete group $G$ ) such that

$$
T_{g} A T_{g}^{-1}=A, \quad g \in G
$$

this means that each $T_{g}$ generates an automorphism $\hat{T}_{g}$ of the algebra $A$ by the formula

$$
\hat{T}_{g}(a)=T_{g} a T_{g}^{-1}, \quad a \in A .
$$

The goal of the paper is to describe the structure of such algebras.

In the Hilbert case (i.e., in the theory of $C^{*}$-algebras), similar objects are closely related to crossed products (see, e.g., [1), and the description of the structure of such algebras has been the subject of numerous studies. In particular, 2] contains necessary and sufficient conditions (in terms of duality theory) for a $C^{*}$-algebra to be isomorphic to a crossed product (of an algebra and a locally compact automorphism group). In the case of a discrete group, conditions for a $C^{*}$-algebra to be isomorphic to a crossed product were found in [3, Chapter 1] (see also Subsection 2.7 in this paper). These conditions are expressed in terms of the group action (the so-called topologically free action), as well as in terms of a certain inequality (Property $(*)$; see (2.3) of this paper) that guarantees the existence of an expectation from the algebra $B\left(A, T_{g}\right)$ to the algebra $A$ (see (2.4), (2.5)).

One of the main problems discussed in the paper is to study whether or not these properties (topologically free action, Property $(*)$, and the dual action of the group) allow us to determine the structure of the algebra $B\left(A, T_{g}\right)$ up to isomorphism in the general Banach case.

In Sections 2 and 3 we study relations of these properties. We also study the naturally occurring Property (**); see 2.3 (reconstruction of an element in $B\left(A, T_{g}\right)$ from its "Fourier coefficient"). In particular, in these sections we show that in many cases these properties are equivalent.

Since in the general Banach case we do not have a universal object similar to the crossed product in the Hilbert case, to describe the structure of the algebra $B\left(A, T_{g}\right)$ we

2000 Mathematics Subject Classification. Primary 46L05. 
need to specify the type of the algebra $A$ and of the isometries $T_{g}$. Sections $4-13$ are devoted to applications of the results obtained in Sections 2, 3 to the description of the structure of particular Banach algebras associated with automorphisms, namely algebras generated by "weighted composition operators" acting both in spaces of continuous functions and in Lebesgue spaces.

The examples described are divided into two classes: operators with continuous coefficients and operators with measurable coefficients. Examples of the first class are studied in Sections 4-7, those of the second class in Sections 9-11. Section 8 serves as a "bridge"

used to pass from the continuous case to the measurable one. We prove that a natural "measurable" replacement of a topologically free action is a metrically free action (see 8.2). We also study relations between these notions; in particular, we show that from a certain point of view these notions are equivalent. This allows us to "transfer" main structural results from the already studied continuous case to the measurable case.

In analyzing these examples we obtain a series of results that establish that the algebras under consideration are isomorphic. Simultaneously, we show that certain features of these algebras are different. In particular, when considering operators in $C(X, E), L_{\mu}^{\infty}(X, E)$, and $L_{\mu}^{1}(X, E)$ we can compute their norms (Theorems 4.2, 6.2, 7.3, 10.2. and 11.2). At the same time, for operators in $L_{\mu}^{p}(X, E), 1<p<\infty$, this cannot be done. Moreover, to obtain theorems about isomorphisms for algebras of type $B\left(A, T_{g}\right)$ in the spaces $C(X, E), L_{\mu}^{\infty}(X, E)$, and $L_{\mu}^{1}(X, E)$ we do not need any information about the structure of the group of automorphism generating operators, whereas this structure (more precisely, the amenability of the group $G$ ) is very important in the study of operators in $L_{\mu}^{p}(X, E), 1<p<\infty$ (Theorems 5.7, 9.4 and Section 13).

In this paper, we will use the following notation. For a topological space $X$ and a normed vector space $B$, we denote by $C(X, B)$ the normed space of all continuous functions on $X$ with values in $B$ endowed with the sup norm. For $X$ we will take only completely regular spaces. By $D, E$, and $F$ we denote various Banach spaces, and by $L(E)$ the Banach algebra of all linear operators in $E$.

\section{2. $B\left(A, T_{g}\right)$. Properties $(*)$ and $(* *)$. Topologically free action}

In this section we introduce and study a number of important properties of algebras associated with automorphisms.

2.1. Let $B\left(A, T_{g}\right)$ be the Banach algebra generated by a Banach algebra $A$ of bounded linear operators acting in a Banach space $D$ and by the group $\left\{T_{g}\right\}_{g \in G}$ of isometries of $D$ (so that $G \ni g \rightarrow T_{g}$ is a representation of a discrete group $G$ ) such that

$$
T_{g} A T_{g}^{-1}=A, \quad g \in G .
$$

Condition (2.1) means that the operator $T_{g}$ generates an automorphism $\hat{T}_{g}$ of the algebra $A$ by the formula

$$
\hat{T}_{g}(a)=T_{g} a T_{g}^{-1}, \quad a \in A .
$$

Examples of algebras $A$, spaces $D$, and operators $T_{g}$ for which we will construct our theory will be given later.

In the case where $A$ is a unital $C^{*}$-subalgebra of the algebra $L(H)$ of operators in a Hilbert space $H$, and $\left\{T_{g}\right\}_{g \in G}$ is a unitary representation of a group $G$ in $H$, by $C^{*}\left(A, T_{g}\right)$ we will denote the $C^{*}$-algebra generated by $A$ and $\left\{T_{g}\right\}_{g \in G}$. 
2.2. Property $(*)$. In the case of $C^{*}$-algebras, it is established in [3, Chapter 2] that one of the most important properties of the algebra $C^{*}\left(A, T_{g}\right)$ allowing us to develop a useful deep theory of such objects is the following

Property $(*)$ : For each finite sum $b=\sum a_{g} T_{g}, a_{g} \in A$, the following inequality holds:

$$
\|b\|=\left\|\sum a_{g} T_{g}\right\| \geq\left\|a_{e}\right\|
$$

here $e$ the unit of the group $G$.

One of the goals of this paper is to ascertain the significance of Property $(*)$ in the Banach case, i.e., to study this property in the algebra $B\left(A, T_{g}\right)$.

If the algebra $B\left(A, T_{g}\right)$ possesses Property $(*)$, then for each $g_{0} \in G$ we can define the mapping (conditional expectation)

$$
N_{g_{0}}: \sum a_{g} T_{g} \rightarrow a_{g_{0}}
$$

which can be extended to a mapping

$$
N_{g_{0}}: B\left(A, T_{g}\right) \rightarrow A .
$$

2.3. Property $(* *)$. Another important property is the following one.

We say that the algebra $B\left(A, T_{g}\right)$ (already possessing Property $\left.(*)\right)$ possesses also Property (**) if

$$
B\left(A, T_{g}\right) \ni b=0 \quad \Leftrightarrow \quad\left\{N_{g}(b)=0 \text { for each } g \in G\right\},
$$

where $N_{g}$ is the mapping introduced earlier.

2.4. Remark. In the case of a $C^{*}$-algebra we have the following result (see $[3$, Theorems 12.8 and 12.4]):

If $G$ a discrete amenable group and the algebra $C^{*}\left(A, T_{g}\right)$ possesses Property $(*)$, then it possesses Property $(* *)$ as well.

In fact, Properties $(*)$ and $(* *)$ allow us to "reconstruct" an element $b \in B\left(A, T_{g}\right)$ from its "Fourier coefficients" $N_{g}(b), g \in G$. Later we will establish that in many natural cases such "reconstruction" can be successfully performed.

Let us recall that in the case of a $C^{*}$-algebra there exists a close connection between Property $(*)$ and the so-called topological freeness of the action of the automorphism group $\left\{\hat{T}_{g}\right\}$ (see [3, Subsection 12.13 and Theorem 12.14]). Therefore it is natural to analyze the relation between similar properties of the algebra $B\left(A, T_{g}\right)$ as well. This will be done in the remaining part of this section.

2.5. First we make several remarks clarifying the further choice of objects and definitions.

Let us note that if $\left\{T_{g}\right\}_{g \in G}$ is a group of isometries satisfying (2.1), then, clearly,

$$
T_{g} \mathbf{Z}(A) T_{g}^{-1}=\mathbf{Z}(A)
$$

where $\mathbf{Z}(A)$ is the center of $A$.

Let $A$ be a Banach algebra isomorphic to $C(X, B)$, where $X$ is a completely regular space and $B$ is a Banach algebra. Then

$$
\mathbf{Z}(A)=C(X, \mathbf{Z}(B)) .
$$

Indeed, the inclusion $\mathbf{Z}(A) \supset C(X, \mathbf{Z}(B))$ is clear and if $\bar{a} \in \mathbf{Z}(A)$, then for each $a \in A$ and each $x_{0} \in X$ we have $\bar{a}\left(x_{0}\right) a\left(x_{0}\right)=a\left(x_{0}\right) \bar{a}\left(x_{0}\right)$. Therefore, choosing $a(x) \equiv b \in B$, we conclude that $\bar{a}\left(x_{0}\right) \in \mathbf{Z}(B)$, i.e., $\mathbf{Z}(A) \subset C(X, \mathbf{Z}(B))$.

For a moment, let us assume that

$$
\mathbf{Z}(B) \cong C(M)
$$


(of course, this assumption is rather restrictive; however, it allows us to clarify a certain idea). Then

$$
\mathbf{Z}(A)=C(X, \mathbf{Z}(B)) \cong C(X, C(M)) \cong C(X \times M) .
$$

If $X$ and $M$ are compact spaces (in this case $X \times M$ is compact as well), then (2.9) and (2.7) mean that the automorphisms $\hat{T}_{g}$ (see (2.2) $)$ generate homeomorphisms $t_{g}$ : $X \times M \rightarrow X \times M$ by the formula

$$
\left[\hat{T}_{g}(z)\right](y)=z\left(t_{g}^{-1}(y)\right), \quad z \in \mathbf{Z}(A), y \in X \times M .
$$

It is clear that if $M=\{\cdot\}$ consists of one point (i.e., $\mathbf{Z}(B)=\{c I\}$, where $I$ is the unit of $B)$, then $X \times M \cong X$ and $t_{g}$ are homeomorphisms of $X$.

2.6. The above arguments allow us to consider only the case where

$$
\mathbf{Z}(B)=\{c I\}
$$

in other words, we will work with the "initial" space $X$ and will not involve additional objects like $X \times M$, etc.

It is clear that if $B=L(E)$, then (2.11) holds.

So, let $A \subset L(D)$ be a Banach algebra of operators isomorphic to $C(X, L(E))$, where $X$ is a completely regular space and $E$ and $D$ are Banach spaces (in this case $\mathbf{Z}(A) \cong$ $C(X)$ ). Let $\left\{T_{g}\right\}_{g \in G}$ be a group of isometries satisfying (2.1). According to (2.7), the automorphisms $\hat{T}_{g}$ in (2.2) preserve the center. In further considerations, we will assume that the action on the center has the form

$$
\left[\hat{T}_{g}(z)\right](x)=z\left(t_{g}^{-1}(x)\right), \quad z \in \mathbf{Z}(A), x \in X,
$$

where $t_{g}: X \rightarrow X$ are some homeomorphisms of $X$.

Remark. Since $\mathbf{Z}(A) \cong C(X) \cong C(\bar{X})$ where $\bar{X}$ is the Stone-Čech compactification of the space $X$, the automorphisms $\hat{T}_{g}$ generate the homeomorphisms $\tau_{g}: \bar{X} \rightarrow \bar{X}$ by the formula

$$
\left[\hat{T}_{g}(z)\right](x)=z\left(\tau_{g}^{-1}(x)\right), \quad x \in \bar{X}
$$

One has to note that in the general case the homeomorphisms $\tau_{g}$ do not preserve the subset $X \subset \bar{X}$.

Example. Let $Y$ be a noncompact completely regular space and $\bar{Y}$ its Stone-Cech compactification. Consider the space $X=Y \sqcup \bar{Y}$, where $\sqcup$ denotes the disjoint union of spaces. It is clear that $C(X) \cong C(\bar{X})=C(\bar{Y} \sqcup \bar{Y})$. There exists a one-to-one correspondence between elements of the algebra $A=C(X)$ and pairs $\left(a_{1}, a_{2}\right)$, where $a_{i} \in C(\bar{Y}), i=1,2$. Let $\hat{T}$ be an automorphism of the algebra $A=\mathbf{Z}(A)$ of the form $\hat{T}\left(a_{1}, a_{2}\right)=\left(a_{2}, a_{1}\right)$. It is clear that this automorphism generates a homeomorphism $\tau$ of the space $\bar{X}$, which does not preserve $X$.

This means that assuming the condition (2.12) to be satisfied, we restrict ourselves to the consideration of the case where the $\tau_{g}$ preserve $X$ (and in this case their restrictions to $X$ concide with $t_{g}$ ).

We made this assumption just to simplify the exposition and not to use $\bar{X}$ instead of $X$. 
2.7. Topologically free action. Denote by $X_{g}, g \in G$, the set

$$
X_{g}=\left\{x \in X: t_{g}(x)=x\right\} .
$$

We say that the group $G$ acts topologically freely on $A$ by automorphisms $\hat{T}_{g}$ (or on $X$ by homeomorphisms $t_{g}$ defined in 2.6) if for each $g \in G, g \neq e$, the set $X_{g}$ has empty interior.

Let us note also that $G$ acts topologically freely if and only if for each finite set $\left\{g_{1}, \ldots, g_{n}\right\} \subset G, g_{i} \neq e$, the set $\left[\bigcup_{i=1}^{n} X_{g_{i}}\right]$ has empty interior.

Similarly to $\left[3,12.13\right.$ and $\left.12.13^{\prime}\right]$ one can show that this definition is equivalent to the following: an action of the group $G$ is topologically free if for each finite set $\left\{g_{1}, \ldots, g_{k}\right\} \subset$ $G$ and each nonempty open set $U \subset X$ there exists a point $x \in U$ such that all points $t_{g_{i}}(x), i=1, \ldots, k$, are distinct.

Since the space $X$ is Hausdorff, the latter definition is equivalent to the following one: the action of a group $G$ is topologically free if for each finite set $\left\{g_{1}, \ldots, g_{k}\right\} \subset G$ and each nonempty open set $U \subset X$ there exists an open set $V \subset U$ such that

$$
t_{g_{i}}(V) \cap t_{g_{j}}(V)=\varnothing, \quad i, j \in\{1, \ldots, k\}, i \neq j .
$$

The next theorem is an analogue of Theorem 12.14 in [3].

2.8. Theorem. If $G$ acts topologically freely, then $B\left(A, T_{g}\right)$ possesses Property (*).

Proof. Consider an arbitrary element $b \in B\left(A, T_{g}\right)$ of the form

$$
b=\sum_{g \in K} a_{g} T_{g},
$$

where $K=\left\{g_{1}, \ldots, g_{n}\right\}$. Choose $\varepsilon>0$ and let $x_{0} \in X$ be a point satisfying the condition

$$
\left\|a_{e}\left(x_{0}\right)\right\|>\left\|a_{e}\right\|-\varepsilon
$$

(such a point $x_{0}$ exists because $A \cong C(X, L(E))$ ). Consider a neighborhood $U$ of the point $x_{0}$ such that

$$
\left\|a_{e}(x)\right\|>\left\|a_{e}\left(x_{0}\right)\right\|-\varepsilon, \quad x \in U .
$$

Since the action of $G$ is topologically free, there exists (see 2.7) a nonempty open set $V$ such that

$$
V \subset U \quad \text { and } \quad t_{g_{i}}^{-1}(V) \cap t_{g_{j}}^{-1}(V)=\varnothing, \quad g_{i}, g_{j} \in K, g_{i} \neq g_{j} .
$$

Let $c \in \mathbf{Z}(A)$ be an element of the form $c=c(x) I$, where $c(\cdot)$ is a continuous function on $X$ such that

(i) $0 \leq c(x) \leq 1$;

(ii) $c\left(x^{\prime}\right)=1$ for some $x^{\prime} \in V$;

(iii) $c(x)=0$ if $x \notin V$.

By (i), (ii), (2.15), (2.16), and the choice of $x^{\prime}$ we have

$$
\left\|c^{2} a_{e}\right\| \geq\left\|a_{e}\left(x^{\prime}\right)\right\|>\left\|a_{e}\right\|-2 \varepsilon
$$

and

$$
\|c b c\| \leq\|b\| .
$$

Moreover, (2.17) and (iii) imply

$$
c \hat{T}_{g}(c)=0, \quad g \neq e, g \in K
$$


Now (2.20), (2.19), and (2.18) imply

$$
\begin{aligned}
\|b\| \geq\|c b c\| & =\left\|c\left(\sum a_{g} T_{g}\right) c\right\|=\left\|\sum c a_{g} \hat{T}_{g}(c) T_{g}\right\| \\
& =\left\|\sum c \hat{T}_{g}(c) a_{g} T_{g}\right\|=\left\|c^{2} a_{e}\right\|>\left\|a_{e}\right\|-2 \varepsilon,
\end{aligned}
$$

and since $\varepsilon$ is arbitrary, the proof is completed.

\section{Properties $(*)$ And $(* *)$, And the dual action of the Group}

As we have already noted (Remark 2.4), in the $C^{*}$-algebra case Property $(* *)(2.6)$ is a consequence of Property $(*)$. In fact, the main reason for this is the following fact (see [3. Theorem 12.8]):

Property $(*)$ implies the isomorphism

$$
C^{*}\left(A, T_{g}\right) \cong A \times_{\hat{T}} G,
$$

where $A \times_{\hat{T}} G$ denotes the crossed product of the algebra $A$ and the group $\left\{\hat{T}_{g}\right\}_{g \in G}$ of automorphisms of $A$ (here $G$ is viewed as a discrete group).

Since in the Banach case there is nothing like the above isomorphism, Property $(* *)$ needs a verification even if the algebra $B\left(A, T_{g}\right)$ possesses Property $(*)$. In the general case (i.e., for an arbitrary discrete group of isometries $\left\{T_{g}\right\}_{g \in G}$ satisfying the condition $T_{g} A T_{g}^{-1}=A$ ) the verification of Property $(* *)$ can be rather complicated. Theorem 3.2 below shows that for a locally compact commutative group $G$ and under an additional assumption (which, as we will see later, is not too restrictive, and, moreover, occurs quite often) the algebra $B\left(A, T_{g}\right)$ possesses both Property $(*)$ and Property $(* *)$.

Before passing to this theorem, let us recall the Kronecker Approximation Theorem that relates continuous and disrete characters of locally simple commutative groups.

3.1. Theorem [4, (26.15)]. Let $G$ be a locally compact commutative group, $\hat{G}$ the dual group, and $\Gamma$ a subgroup of $G$. Next, let $f$ be an arbitrary (not necessarily continuous) character of the group $\Gamma$ and $g_{1}, \ldots, g_{n}$ elements of $\Gamma$. Then for each $\varepsilon>0$ there exists a continuous character $\chi \in \hat{G}$ such that

$$
\left|f\left(g_{i}\right)-\chi\left(g_{i}\right)\right|<\varepsilon, \quad i=1, \ldots, n .
$$

3.2. Theorem. Let $G$ be a locally compact commutative group such that for each finite set $K \subset G$ and an arbitrary character $\chi \in \hat{G}$ we have

$$
\left\|\sum_{g \in K} a_{g} T_{g}\right\|=\left\|\sum_{g \in K} a_{g} \chi(g) T_{g}\right\| .
$$

Then the algebra $B\left(A, T_{g}\right)$ possesses Properties $(*)$ and $(* *)$.

Proof. By the previous theorem, if (3.1) holds for each $\chi \in \hat{G}$, then it also holds for an arbitrary (not necessarily continuous) character of $G$ (i.e., for a character of the group $G$ regarded as a discrete group). Therefore below we assume that $G$ is a discrete group and denote by $\hat{G}$ the corresponding dual group.

Let $b \in B\left(A, T_{g}\right)$ be an element of the form

$$
b=\sum_{g \in K} a_{g} T_{g}, \quad|K|<\infty .
$$

By $b(\chi), \chi \in \hat{G}$, we denote the element of $B\left(A, T_{g}\right)$ defined by the formula

$$
b(\chi)=\sum_{g \in K} a_{g} \chi(g) T_{g} .
$$


Equality (3.1) means that

$$
\|b\|=\|b(\chi)\|, \quad \chi \in \hat{G} .
$$

First let is prove that this condition implies Property $(*)$.

Let us recall that for each $c \in L(F)$ we have

$$
\|c\|=\sup _{\substack{\xi \in F,\|\xi\|=1 \\ \eta \in F^{*},\|\eta\|=1}}|\langle c \xi, \eta\rangle| ;
$$

here $F^{*}$ is the space dual to $F$ and $\langle\xi, \eta\rangle=\eta(\xi)$ ( $F$ is the space where the operators of $B\left(A, T_{g}\right)$ act $)$.

Choose $\varepsilon>0$ and let $\xi_{0} \in F$ and $\eta_{0} \in F^{*},\left\|\xi_{0}\right\|=\left\|\eta_{0}\right\|=1$, be vectors such that

$$
\left\|a_{e}\right\| \geq\left|\left\langle a_{e} \xi_{0}, \eta_{0}\right\rangle\right|>\left\|a_{e}\right\|-\varepsilon
$$

where $e$ is the unit of $G$.

Formulas (3.2) and (3.3) imply that

$$
\begin{aligned}
\|b\| & =\|b(\chi)\| \geq\left|\left\langle b(\chi) \xi_{0}, \eta_{0}\right\rangle\right|=\left|\left\langle\sum_{K} a_{g} \chi(g) T_{g} \xi_{0}, \eta_{0}\right\rangle\right| \\
& =\left|\sum_{K}\left\langle a_{g} T_{g} \xi_{0}, \eta_{0}\right\rangle \chi(g)\right|=\left|\sum_{K} \alpha_{g} \chi(g)\right|,
\end{aligned}
$$

where $\alpha_{g}=\left\langle a_{g} T_{g} \xi_{0}, \eta_{0}\right\rangle$.

Consider a function $f(\chi) \in C(\hat{G})$ of the form

$$
f(\chi)=\sum_{K} \alpha_{g} g(\chi)
$$

where $g(\chi)=\chi(g)$ is a character of $\hat{G}$. Since the group $G$ is discrete, $\hat{G}$ is compact 4 , (23.17)] and the Fourier coefficient $\alpha_{e}$ of the function $f$ can be computed [4, (23.19)] by the formula

$$
\alpha_{e}=\int_{\hat{G}} f(\chi) d \mu
$$

( $\mu$ is the normalized Haar measure on $\hat{G}$ ). In turn, this implies (according to the definition of $f$ and to (3.5))

$$
\left|\alpha_{e}\right| \leq \int_{\hat{G}}|f(\chi)| d \mu \leq \max _{\hat{G}}|f(\chi)| \leq\|b\| .
$$

Together with (3.4) this means that

$$
\|b\| \geq\left\|a_{e}\right\|-\varepsilon
$$

Therefore (since $\varepsilon$ is arbitrary) Property $(*)$ is proved.

Now let us verify Property $(* *)$.

Let $b$ be an arbitrary element in $B\left(A, T_{g}\right)$.

If (3.2) holds, then, as we have already verified, the algebra $B\left(A, T_{g}\right)$ possesses Property $(*)$. Therefore, the mapping $N_{g_{0}}$ given by formula (2.5) is well defined. We need to show that if $N_{g}(b)=0$ for each $g \in G$, then $b=0$.

Clearly, it suffices to prove that for each $\chi \in F$ and $\eta \in F^{*}$ with $\|\xi\|=\|\eta\|=1$ we have

$$
\langle b \xi, \eta\rangle=0 .
$$

Let $\left\{b_{n}\right\}$ be a sequence of elements $B\left(A, T_{g}\right)$ of the form

$$
b_{n}=\sum_{K_{n}} a_{g}^{(n)} T_{g}, \quad\left|K_{n}\right|<\infty
$$


converging to $b$. Consider the elements $b_{n}(\chi), \chi \in \hat{G}$, defined by the formula

$$
b_{n}(\chi)=\sum_{K_{n}} a_{g}^{(n)} \chi(g) T_{g}
$$

and the sequence $\left\{f_{n}(\chi)\right\}$ of functions (continuous on $\hat{G}$ ) of the form

$$
f_{n}(\chi)=\left\langle b_{n}(\chi) \xi, \eta\right\rangle=\sum_{K_{n}}\left\langle a_{g}^{(n)} T_{g} \xi, \eta\right\rangle \chi(g)=\sum_{K_{n}} \alpha_{g}^{(n)} \chi(g),
$$

where $\alpha_{g}^{(n)}=\left\langle a_{g}^{(n)} T_{g} \xi, \eta\right\rangle$. Since $b_{n} \rightarrow b$ as $n \rightarrow \infty$, we have (by (3.2))

$$
\left\|b_{n_{1}}(\chi)-b_{n_{2}}(\chi)\right\|=\left\|b_{n_{1}}-b_{n_{2}}\right\|_{n_{1}, n_{2} \rightarrow \infty}^{\longrightarrow} 0 .
$$

Therefore, for each $\chi_{0} \in \hat{G}$, the sequence $\left\{b_{n}\left(\chi_{0}\right)\right\}$ converges to an element $b\left(\chi_{0}\right) \in$ $B\left(A, T_{g}\right)$.

Let $f(\chi)$ be a function of the form

$$
f(\chi)=\langle b(\chi) \xi, \eta\rangle .
$$

Using (3.9) we obtain

$$
\left|f_{n_{1}}(\chi)-f_{n_{2}}(\chi)\right|=\left|\left\langle\left[b_{n_{1}}(\chi)-b_{n_{2}}(\chi)\right] \xi, \eta\right\rangle\right| \leq\left\|b_{n_{1}}-b_{n_{2}}\right\|_{n_{1}, n_{2} \rightarrow \infty}^{\longrightarrow} 0 .
$$

Therefore, the sequence $\left\{f_{n}\right\}$ of (continuous) functions converges uniformly (on $\hat{G}$ ) to $f$. Therefore, $f$ is a continuous function and (since $\mu(\hat{G})=1) f \in L^{2}(\hat{G})$.

Let

$$
f(\chi)=\sum_{G} \alpha_{g} g(\chi)
$$

where in the right-hand side we have the Fourier series for $f$. Since $f_{n} \rightarrow f\left(\right.$ in $L^{2}(\hat{G})$ ), we have

$$
\alpha_{g}^{(n)} \rightarrow \alpha_{g} \text { for each } g \in G,
$$

where $\alpha_{g}^{(n)}$ are defined in (3.8). Now let us note that Property $(*)$ implies that

$$
\left\|a_{g}^{(n)}\right\| \rightarrow\left\|N_{g}(b)\right\| \text { for each } g \in G .
$$

Let us also note that

$$
\left|\alpha_{g}^{(n)}\right|=\left|\left\langle a_{g}^{(n)} T_{g} \xi, \eta\right\rangle\right| \leq\left\|a_{g}^{(n)}\right\| .
$$

Together with (3.10) and (3.11) this means that

$$
\alpha_{g}=0 \text { for each } g \in G .
$$

In turn, formula (3.12) and the continuity of $f$ imply that

$$
f(\chi)=0 \text { for each } \chi \in \hat{G} \text {. }
$$

In particular,

$$
f(1)=\langle b \xi, \eta\rangle=0 .
$$

Therefore (3.7) holds and the proof is completed. 
3.3. Remark. (1) The theorem just proved shows that (in the case where the group $G$ is commutative) property (3.1) implies Properties $(*)$ and $(* *)$. On the other hand, in the $C^{*}$-algebra case Property $(*)$ implies (3.1). Indeed, by [3, Theorem 12.8], if $C^{*}\left(A, T_{g}\right)$ possesses Property $(*)$, then

$$
C^{*}\left(A, T_{g}\right) \cong A \times_{\hat{T}} G
$$

and the isomorphism is established by the mapping

$$
\sum a_{g} T_{g} \longleftrightarrow \sum a_{g} \otimes g
$$

For a given character $\chi \in \hat{G}$ define the representation

$$
\pi_{\chi}: A \times_{\hat{T}} G \rightarrow A \times_{\hat{T}} G
$$

by the formula

$$
\pi_{\chi}\left(\sum a_{g} \otimes g\right)=\sum \chi(g) a_{g} \otimes g
$$

Then

$$
\left\|\pi_{\chi}\left(\sum a_{g} \otimes g\right)\right\|=\left\|\sum a_{g} \otimes g\right\|
$$

(this again follows from [3, Theorem 12.8 and remarks]), so that $\pi_{\chi}\left(A \times_{\hat{T}} G\right)$ possesses Property (*) since $A \times_{\hat{T}} G$ possesses it (see [3, 12.3]). Now (3.15), together with (3.14) and (3.13), means that

$$
\left\|\sum a_{g} \chi(g) T_{g}\right\|=\left\|\sum a_{g} T_{g}\right\|
$$

and (3.1) holds.

Therefore, in the $C^{*}$-algebra case (and in the case where $G$ is commutative and locally compact), Properties $(*)$ and (3.1) are equivalent. Therefore in the general case one can view (3.1) as a certain substitute for Property $(*)$ (under which Properties $(*)$ and $(* *)$ hold simultaneously, as in the $C^{*}$ case).

(2) One should note that if $G$ is not commutative, one has to consider conditions different from (3.1) (in this regard, see, e.g., [3, Lemma 22.6]).

(3) Let us note also that whereas in Theorem 2.8 we assumed that the algebra $A$ is of a special form (described in 2.6), in Theorem $3.2 A$ is an arbitrary Banach algebra.

Now we present an example of an algebra $B\left(A, T_{g}\right)$ for which the verification of property (3.1) is easy.

3.4. Example. Let $X=G$ be a locally compact commutative group, $A$ an algebra of operators that is isomorphic to $C(X, L(E))$, and $\left\{T_{g}\right\}_{g \in G}$ a group of isometries satisfying (2.1) and such that

$$
\left(T_{g} a T_{g}^{-1}\right)(x)=a(x \cdot g), \quad a \in A .
$$

In this case,

The algebra $B\left(A, T_{g}\right)$ generated by $A$ and $\left\{T_{g}\right\}_{g \in G}$ satisfies (3.1).

Indeed, for each $\chi \in \hat{G}$ consider the operator $\bar{\chi} \in \mathbf{Z}(A)$ corresponding to the operatorvalued function $\chi(x) I$ ( $I$ is the identity operator in $E$ ). It is clear that $\bar{\chi}$ is an invertible operator and for each operator

$$
b=\sum_{g \in K} a_{g} T_{g}, \quad|K|<\infty,
$$

we have

$$
\|b\|=\left\|\bar{\chi}^{-1} b \bar{\chi}\right\|=\left\|\sum a_{g} \chi(g) T_{g}\right\| .
$$

Therefore, (3.1) is satisfied. 
3.5. Remark. In fact, we did not use that $G$ is commutative and a similar result can be proved for an arbitrary topological group.

3.6. Example. Regular representation of an algebra and a group of automorphisms. Let us consider one more example where Properties $(*)$ and $(* *)$ can be easily verified, namely the regular representation of an algebra $A$ and a group of automorphisms $\left\{\hat{T}_{g}\right\}_{g \in G}$.

Let $A \subset L(D)$ be a Banach algebra and $\left\{\hat{T}_{g}\right\}_{g \in G}$ a group of automorphisms of $A$ (here $G$ is an arbitrary group, not necessarily commutative).

By $H$ we will denote any of the spaces $l^{p}(G, D), 1 \leq p \leq \infty$, or $l_{0}(G, D)$ (here $l_{0}(G, D)$ is the space of vector-valued functions on $G$ with values in $D$ that vanish at infinity, with the sup norm). Define the operators $V_{g_{0}}: H \rightarrow H$ by the formula

$$
\left(V_{g_{0}} \xi\right)(g)=\xi\left(g g_{0}\right), \quad g, g_{0} \in G,
$$

and consider the isomorphic to $A$ (as a Banach algebra) algebra $\bar{A} \subset L(H)$ of operators defined by the formula

$$
(\bar{a} \xi)(g)=\hat{T}_{g}(a) \xi(g), \quad a \in A .
$$

Direct computations show that (in this notation) we have

$$
V_{g} \bar{a} V_{g}^{-1}=\overline{\hat{T}_{g}(a)},
$$

which means (since $A$ and $\bar{A}$ are isomorphic) that the operators $V_{g}, g \in G$, defined by (3.16) yield automorphisms $\hat{T}_{g}$ of the algebra $\bar{A}$.

The algebra $B\left(\bar{A}, V_{g}\right) \subset L(H)$ is called the (right) regular representation corresponding to the algebra $A$ and the group of automorphisms $\left\{\hat{T}_{g}\right\}_{g \in G}$ (in fact, we have a family of representations depending on the choice of a specific space $H$ ).

Let us note that

The algebra $B\left(\bar{A}, V_{g}\right)$ possesses Properties $(*)$, (**), and (3.1) (for each space $H$ ).

First, let us verify Property $(*)$.

Take an operator $b$ of the form

$$
b=\sum_{g \in K} \bar{a} V_{g}, \quad|K|<\infty .
$$

Choose an arbitrary $\varepsilon>0$ and take a vector $\eta \in D,\|\eta\|=1$, such that

$$
\left\|a_{e} \eta\right\| \geq\left\|a_{e}\right\|-\varepsilon \text {. }
$$

Let $\xi \in H$ be the vector given by the formula

$$
\xi(g)=\delta(e, g) \eta,
$$

where $\delta(e, g)$ is the Kronecker symbol. By the definition of $\xi$ and the explicit formula for $b$ we have

$$
\|b \xi\|=\left\|\sum_{g \in K} \bar{a} V_{g}(\xi)\right\| \geq\left\|\bar{a}_{e} \xi\right\|=\left\|a_{e} \eta\right\| \geq\left\|a_{e}\right\|-\varepsilon=\left\|\bar{a}_{e}\right\|-\varepsilon,
$$

which (since $\varepsilon$ is arbitrary) proves Property $(*)$.

Now let us verify Property $(* *)$.

We begin with the case where $H$ is one of the spaces $l^{p}(G, D), 1 \leq p<\infty$, or $l_{0}(G, D)$. In this case an operator $b \in L(H)$ is determined by its matrix

$$
[b]_{h, g}=\hat{T}_{h}\left[N\left(b V_{g^{-1} h}\right)\right], \quad h, g \in G,
$$

(the mapping $N: B\left(\bar{A}, V_{g}\right) \rightarrow A \cong \bar{A}$ is defined by (2.5) and depends on the choice of an isomorphism between $A$ and $\bar{A}$ ). 
Since an operator is the zero operator if and only if its matrix is zero, Property $(* *)$ is proved in the case under consideration.

It remains to consider the case $H=l^{\infty}(G, D)$.

Here we first note that for an arbitrary operator $b$ of the form (3.18) we have

$$
\|b\|_{l^{\infty}(G, D)}=\|b\|_{l_{0}(G, D)} .
$$

Indeed, since $l_{0}(G, D) \subset l^{\infty}(G, D)$, we have

$$
\|b\|_{l^{\infty}(G, D)} \geq\|b\|_{l_{0}(G, D)} .
$$

To obtain the opposite inequality, choose $\varepsilon>0$ and consider an arbitrary vector $\xi \in$ $l^{\infty}(G, D)$ such that

$$
\|\xi\|=1
$$

and

$$
\|b \xi\|_{l \infty(G, D)}>\lambda-\varepsilon
$$

where $\lambda=\|b\|_{l^{\infty}(G, D)}$. Property (3.21) means that there exists a point $g_{0} \in G$ such that

$$
\left\|(b \xi)\left(g_{0}\right)\right\|>\lambda-\varepsilon .
$$

Define the vector $\eta \in l_{0}(G, D)$ by the formula

$$
\eta(g)= \begin{cases}\xi(g), & g \in M=\left[\bigcup_{g \in K}\left(g g_{0}\right)\right], \\ 0, & g \notin M .\end{cases}
$$

Formulas (3.20) and (3.22) imply that for this vector $\eta$ we have

$$
\|\eta\|_{l_{0}(G, D)} \leq 1
$$

and

$$
\|b \eta\|_{l_{0}(G, D)}>\lambda-\varepsilon,
$$

which (since $\varepsilon$ is arbitrary) implies that

$$
\|b \eta\|_{l_{0}(G, D)} \geq \lambda=\|b\|_{l^{\infty}(G, D)},
$$

thus proving (3.19).

Since (3.19) holds for all $b$ of the form (3.18), it also holds for an arbitrary $b \in$ $B\left(\bar{A}, V_{g}\right)$. Therefore, since the algebra $B\left(\bar{A}, V_{g}\right)$ acting in $l_{0}(G, D)$ possesses Property $(* *)$, it possesses this property when it acts in $l^{\infty}(G, D)$ as well.

It remains to verify (3.1).

Take a character $\chi \in \hat{G}$ and let $\bar{\chi} \in L(H)$ be an isometry defined by the operatorvalued function $\{\chi(g) I\}_{g \in G}$ (here $I$ is the identity operator in $D$ ). It is clear that $\bar{\chi}$ commutes with each element $\bar{a} \in \bar{A}$, and for an operator $b$ of the form (3.18) we have

$$
\|b\|=\left\|\bar{\chi}^{-1} b \bar{\chi}\right\|=\left\|\sum_{g \in K} \bar{a}_{g} \chi(g) V_{g}\right\|,
$$

as required. 
3.7. Remark. (1) Let us emphasize again that since $G$ was not assumed to be commutative, formula (3.1) does not automatically imply Properties (*) and (**).

(2) Example 3.6 represents a very important (for future considerations) class of algebras (regular representations) possessing Properties $(*)$ and $(* *)$. In fact, the arguments used in this example show that here the main reason for Properties $(*)$ and $(* *)$ to hold is that the regular representation consists of operators acting in discrete spaces $\left(l^{p}(G, D), l_{0}(G, D)\right)$, so that these operators can be defined by their matrices.

More precisely, let $H$ be one of the spaces $l^{p}(G, D), 1 \leq p<\infty$, or $l_{0}(G, D)$, where $D$ is a Banach space. Let $\mathrm{A} \subset L(H)$ be the algebra of the operators of the form

$$
(a \xi)(g)=a(g) \xi(g), \quad \xi \in H, g \in G, a \in \mathrm{A},
$$

where $a(g) \in L(D)$ and $\sup _{G}\|a(g)\|<\infty$ (hence also $\left.\mathrm{A} \cong l^{\infty}(G, L(D))\right)$. Let the operators $\mathrm{\vee}_{g_{0}}: H \rightarrow H$ be given by the formula

$$
\left(\mathrm{V}_{g_{0}} \xi\right)(g)=\xi\left(g g_{0}\right), \quad g, g_{0} \in G .
$$

Consider the algebra $B\left(\mathrm{~A}, \mathrm{~V}_{g}\right) \subset L(H)$. An obvious modification of arguments used in the analysis of $B\left(\bar{A}, V_{g}\right)$ in Example 3.6 shows that

$B\left(\mathrm{~A}, \mathrm{~V}_{g}\right)$ possesses Properties $(*),(* *)$, and (3.1) (for each $H$ mentioned above).

In fact, the algebra $B\left(\mathrm{~A}, \vee_{g}\right)$ "dominates" $B\left(\bar{A}, V_{g}\right)$, so that for each $H$ (mentioned in Example 3.6) we have

$$
\bar{A} \subset \mathrm{A} \text { and } V_{g}=\mathrm{V}_{g},
$$

i.e., $B\left(\bar{A}, V_{g}\right)$ is a subalgebra of $B\left(\mathrm{~A}, \mathrm{~V}_{g}\right)$.

In Sections 4-7 below we present a number of examples of Banach algebras generated by "weighted composition operators" acting in various spaces. We study the structure of these algebras and, in particular, establish that in many natural topological situations topological freeness of an action of a group $G$ implies Property (**) (conditions (3.1)).

In all these cases we will fix not only the type of the algebra $A \subset L(D)$, but also the space $D$ and the explicit form of the operators $T_{g}, g \in G$. The reason is that the norm $\left\|\sum a_{g} T_{g}\right\|$ is computed (estimated) differently in different spaces even if the operators $T_{g}$ generate the same automorphisms of $A$.

\section{Example 1. Operators in $C(X, E)$}

4.1. Let $D=C(X, E)$ be the Banach algebra of all continuous functions on a completely regular space $X$ with values in a Banach space $E$, endowed with the sup norm. Let $A=C(X, L(E)) \subset L(D)$ be the algebra of operators of multiplication by continuous operator-valued functions with the sup norm, i.e.,

$$
(a f)(x)=a(x) f(x)
$$

for $a \in A$ and $f \in D$.

In considering operators of type (4.1) (where $f=\{f(x)\}$ is a vector-valued function and $a=\{a(x)\}$ is an operator-valued function, $a(x) \in L(F), F \ni f(x))$, we will call them multiplication (or diagonal) operators.

Let $\left\{t_{g}\right\}_{g \in G}$ be a group of homeomorphisms of the space $X$. By $T_{g}$ we denote the isometry in $D$ defined by the formula

$$
\left(T_{g} f\right)(x)=f\left(t_{g}^{-1} x\right) .
$$

Clearly, $T_{g}$ satisfies (2.1) and the above homeomorphisms $t_{g}$ coincide with homeomorphisms defined in (2.12). 
For the algebra $B\left(A, T_{g}\right) \subset L(D)$ generated by these $A$ and $T_{g}$, we can prove much more than just the validity of Properties $(*)$ and $(* *)$ and formula (3.1). Namely, let $K \subset G$ be an arbitrary finite set. Denote by $B_{K}(E)$ and $S_{K}(E)$ the sets

$$
\begin{aligned}
& B_{K}(E)=\left\{\left\{f_{g}\right\}_{g \in K}: f_{g} \in E,\left\|f_{g}\right\| \leq 1, g \in K\right\}, \\
& S_{K}(E)=\left\{\left\{f_{g}\right\}_{g \in K}: f_{g} \in E,\left\|f_{g}\right\|=1, g \in K\right\},
\end{aligned}
$$

respectively. We begin the analysis of the algebra $B\left(A, T_{g}\right)$ with the following simple observation.

4.2. Theorem. If $G$ acts topologically freely, then

$$
\left\|\sum_{g \in K} a_{g} T_{g}\right\|=\sup _{X} \sup _{\left\{f_{g}\right\}_{g \in K} \in S_{K}(E)}\left\|\sum_{g \in K} a_{g}(x) f_{g}\right\|=\sup _{X} \sup _{\left\{f_{g}\right\}_{g \in K} \in B_{K}(E)}\left\|\sum_{g \in K} a_{g}(x) f_{g}\right\| .
$$

Proof. To simplify the notation, we will omit the indication to the space $(E)$ in $B_{K}(E)$ and $S_{K}(E)$.

We begin with proving the equality

$$
\left\|\sum_{g \in K} a_{g} T_{g}\right\|=\sup _{X} \sup _{\left\{f_{g}\right\}_{g} \in K \in B_{K}(E)}\left\|\sum_{g \in K} a_{g}(x) f_{g}\right\| .
$$

Let us note that for each $f \in C(X, E)$ with $\|f\|=1$ we have

$$
\left\|\sum_{g \in K} a_{g} T_{g} f\right\|=\sup _{X}\left\|\sum_{g \in K} a_{g}(x) f\left(t_{g}^{-1}(x)\right)\right\|=\sup _{X}\left\|\sum_{g \in K} a_{g}(x) f_{g}(x)\right\|,
$$

where $\left\|f_{g}(x)\right\|=\left\|f\left(t_{g}^{-1}(x)\right)\right\| \leq 1$. Therefore,

$$
\left\|\sum_{g \in K} a_{g} T_{g}\right\| \leq \sup _{X} \sup _{\left\{f_{g}\right\} \in B_{K}}\left\|\sum_{g \in K} a_{g}(x) f_{g}\right\| .
$$

To prove the opposite inequality, choose a point $x_{0} \in X$ and a family $\left\{f_{g}\right\} \in B_{K}$, and let

$$
\left\|\sum_{g \in K} a_{g}\left(x_{0}\right) f_{g}\right\|=\lambda
$$

Since $a_{g} \in C(X, L(E))$, for each $\varepsilon>0$ there exists a neighborhood $U$ of the point $x_{0}$ such that

$$
\left\|\sum_{g \in K} a_{g}(x) f_{g}\right\|>\lambda-\varepsilon \text { for each } x \in U .
$$

Since the action of $G$ is topologically free, we can find a point $x^{\prime} \in U$ and a neighborhood $V \subset U$ of $x^{\prime}$ such that

$$
V \cap t_{g}^{-1}(V)=\varnothing, \quad g \in K, g \neq e .
$$

Choose functions $\phi_{g} \in C(X), g \in K$, such that

$$
\begin{cases}0 \leq \phi_{g}(x) \leq 1, & \\ \phi_{g}\left(t_{g}^{-1}\left(x^{\prime}\right)\right)=1, & g \in K, \\ \phi_{g}\left(X \backslash t_{g}^{-1}(V)\right)=0, & g \in K,\end{cases}
$$

and set

$$
f=\sum_{g \in K} \phi_{g}(x) f_{g} \in C(X, E)
$$


The choice of functions $\phi_{g}$ and the fact that $\left\{f_{g}\right\} \in B_{K}$ show that

$$
\|f\| \leq 1 \text {. }
$$

On the other hand,

$$
\left\|\left(\sum a_{g} T_{g} f\right)\left(x^{\prime}\right)\right\|=\left\|\sum a_{g}\left(x^{\prime}\right) f_{g}\right\| \geq \lambda-\varepsilon,
$$

where the latter inequality follows from (4.8). Now the definition of $\lambda$ (see (4.7)), together with the arbitrariness of $\varepsilon$ and (4.12) implies that inequality (4.13) means

$$
\left\|\sum a_{g} T_{g}\right\| \geq \sup _{X} \sup _{\left\{f_{g}\right\} \in B_{K}}\left\|\sum_{g \in K} a_{g}(x) f_{g}\right\|,
$$

completing the proof of (4.6).

Now we note that (4.5) follows from (4.6). Indeed, consider the space

$$
\tilde{E}=E_{1} \times E_{2} \times \cdots \times E_{|K|}, \quad E_{i}=E
$$

with the norm

$$
\|\tilde{e}\|_{\tilde{E}}=\left\|\left(e_{1}, \ldots, e_{|K|}\right)\right\|_{\tilde{E}}=\max _{i \in\{1, \ldots,|K|\}}\left\|e_{i}\right\|_{E}
$$

and the operator $\tilde{b}(x) \in L(\tilde{E}, E)$ defined by the formula

$$
\tilde{b}(x)\left(\left(e_{1}, \ldots, e_{|K|}\right)\right)=a_{g_{1}}(x) e_{1}+\cdots+a_{g_{|K|}}(x) e_{|K|}
$$

$\left(\left\{g_{1}, \ldots, g_{|K|}\right\}=K, x \in X\right)$. It is clear that $B_{K}(E)$ can be identified with the unit ball in $\tilde{E}$ and $B_{K}(E)$ is the convex hull of $S_{K}(E)$.

Since the function

$$
\phi(\tilde{e})=\|\tilde{b}(x)(\tilde{e})\|_{\tilde{E}}
$$

is convex on $\tilde{E}$, the mentioned relation between $B_{K}(E)$ and $S_{K}(E)$ implies the following equalities:

$$
\|\tilde{b}(x)\|=\sup _{\tilde{e} \in B_{K}(E)}\|\tilde{b}(x)(\tilde{e})\|=\sup _{\tilde{e} \in S_{K}(E)}\|\tilde{b}(x)(\tilde{e})\| .
$$

These equalities and the explicit form of $\tilde{b}(x)$ mean that (4.6) and (4.5) are equivalent.

4.3. Remark. (1) If $E=\mathbf{C}$ is the field of complex numbers (i.e., $D=C(X)$ and $A=C(X)$ ), then (4.5) implies the following fact:

If $G$ acts topologically freely, then

$$
\left\|\sum_{K} a_{g} T_{g}\right\|=\sup _{X} \sum_{K}\left|a_{g}(x)\right| .
$$

Indeed,

$$
\sup _{X} \sup _{\left\{f_{g}\right\}_{g \in K} \in S_{K}(\mathbf{C})}\left|\sum a_{g}(x) f_{g}\right| \leq \sup _{X} \sum\left|a_{g}(x)\right|,
$$

and to prove the opposite inequality it suffices to put, for each $x \in X$ and $g \in K$,

$$
f_{g}(x)=\left\{\begin{array}{lll}
{\left[\arg a_{g}(x)\right]^{-1}} & \text { if } & a_{g}(x) \neq 0 \\
1 & \text { if } & a_{g}(x)=0
\end{array}\right.
$$

(2) Formula (4.5) shows, in addition, that

If the action of $G$ is topologically free, then

$$
\left\|\sum_{K} a_{g} T_{g}\right\|=\left\|\tilde{b}_{K}\right\|
$$

where the operator

$$
\tilde{b}_{K}: D_{1} \times D_{2} \times \cdots \times D_{|K|} \rightarrow D, \quad D_{i}=D
$$


is given by the formula

$$
\tilde{b}_{K}\left(\xi_{1}, \ldots, \xi_{|K|}\right)=a_{g_{1}} \xi_{1}+\cdots+a g_{|K|} \xi_{|K|}
$$

$\left(\left\{g_{1}, \ldots, g_{|K|}\right\}=K\right)$.

Now we continue the study of the algebra $B\left(A, T_{g}\right)$ and prove that equality (4.5) implies the following result.

4.4. Lemma. Let $B\left(A, T_{g}\right)$ be the algebra described in 4.1 and $B\left(\bar{A}, V_{g}\right)$ the corresponding regular representation of $A$ and $\left\{\hat{T}_{g}\right\}_{g \in G}$ in the space $H=l_{0}(G, C(X, E)$ ) (or $\left.l^{\infty}(G, C(X, E))\right)$ (see 3.6). If the action of $G$ is topologically free, then the mappings

$$
a \rightarrow \bar{a}, \quad a \in A, \quad T_{g} \rightarrow V_{g}, \quad g \in G
$$

define an isomorphism

$$
B\left(A, T_{g}\right) \cong B\left(\bar{A}, V_{g}\right) .
$$

Proof. Direct computations show that the norm of the element

$$
\bar{b}=\sum_{g \in K} \bar{a}_{g} V_{g} \in B\left(\bar{A}, V_{g}\right)
$$

equals

$$
\sup _{X} \sup _{\left\{f_{g}\right\} \in B_{K}(E)}\left\|\sum_{g \in K} a_{g}(x) f_{g}\right\| .
$$

Now the lemma follows from (4.5).

4.5. Corollary. Let $B\left(A, T_{g}\right)$ be the algebra described in 4.1 and let the action of $G$ be topologically free. Then $B\left(A, T_{g}\right)$ possesses Properties $(*),(* *)$, and (3.1).

Proof. It follows from Lemma 4.4 and Example 3.6.

\section{Example 2. Operators in $L_{\mu}^{p}(X, E), 1<p<\infty$}

5.1. Let $X$ be a completely regular space and $\mu$ a Borel $\sigma$-finite measure on $X$ such that the support of $\mu$ coincides with $X$. Consider the Banach space $D=L_{\mu}^{p}(X, E)$, where $1<p<\infty$ (the cases $p=1$ and $p=\infty$ will be considered separately in Examples 3 and 4).

Let $A=C(X, L(E))$ be the algebra of operators defined by formula (4.1) and let $\left\{t_{g}\right\}_{g \in G}$ be a group of homeomorphisms of $X$ preserving the equivalence class of the measure $\mu$ (i.e., the measure $t_{g}(\mu)$ is absolutely continuous with respect to $\mu$ ). By $T_{g}$ we denote the isometry of $D$ of the form

$$
\left(T_{g} f\right)(x)=\left[\frac{t_{g}^{-1}(\mu)}{d \mu}\right]^{\frac{1}{p}} f\left(t_{g}^{-1}(x)\right),
$$

where $\frac{t_{g}^{-1}(\mu)}{d \mu}$ is the Radon-Nikodým derivative of the measure $t_{g}^{-1}(\mu)$ with respect to the measure $\mu$. Let $B\left(A, T_{g}\right) \subset L(D)$ be the Banach algebra generated by $A$ and $\left\{\hat{T}_{g}\right\}_{g \in G}$.

Our description of the algebra $B\left(A, T_{g}\right)$ is divided into several steps.

First of all, let us introduce representations of the algebra $B\left(A, T_{g}\right)$ related to the trajectories of points of $X$. Namely, for each point $x \in X$ define the representation

$$
\pi_{x}: B\left(A, T_{g}\right) \rightarrow L\left(l^{p}(G, E)\right), \quad \pi_{x}(b)=b_{x}, \quad b \in B\left(A, T_{g}\right)
$$

by the formulas

$$
\begin{aligned}
\left(\pi_{x}(a) \xi\right)_{g} & =a\left(t_{g}^{-1}(x)\right) \xi_{g}, \\
\left(\pi_{x}\left(T_{g_{0}}\right) \xi\right)_{g} & =\xi_{g g_{0}},
\end{aligned}
$$

where $\xi=\left(\xi_{g}\right)_{g \in G} \in l^{p}(G, E)$ and $a \in A$. 
In this notation our first observation is the following

5.2. Lemma. Let $b \in B\left(A, T_{g}\right) \subset L\left(L_{\mu}^{p}(X, E)\right)$ be an operator of the form

$$
b=\sum_{K} a_{g} T_{g}, \quad|K|<\infty
$$

(the algebra $B\left(A, T_{g}\right)$ is defined in 5.1). If the action of $G$ is topologically free, then

$$
\|b\| \geq \sup _{x}\left\|b_{x}\right\|
$$

where the operators $b_{x}$ are defined in (5.2)-(5.4). (In particular, $b \rightarrow b_{x}$ is indeed $a$ representation of $B\left(A, T_{g}\right)$.)

Proof. Choose a point $x \in X$ and $\varepsilon>0$, and let $\eta \in l^{p}(G, E)$ be a vector such that

$$
\|\eta\|=1
$$

and

$$
\left\|b_{x} \eta\right\| \geq\left\|b_{x}\right\|-\varepsilon
$$

where $b$ is defined by (5.5). We can assume that the vector $\eta=\left(\eta_{g}\right)$ has only a finite number of nonzero coordinates $\eta_{g}$, i.e., that there exists a finite set $M \subset G$ such that

$$
\eta_{g}=0, \quad g \notin M .
$$

Let

$$
K_{1}=\bigcup_{g \in K} M \cdot g^{-1}
$$

It is clear that $\left|K_{1}\right|<\infty$.

Since $G$ acts topologically freely, there exists a nonempty open subset $V \subset X$ such that $\mu(V)>0$ and

$$
t_{g_{1}}^{-1}(V) \cap t_{g_{2}}^{-1}(V)=\varnothing, \quad g_{1}, g_{2} \in\left[K_{1} \cup M\right], g_{1} \neq g_{2},
$$

and for each $g_{0} \in K$ and $g_{1} \in K_{1}$ we have

$$
\left\|a_{g_{0}}\left(t_{g_{1}}^{-1}(x)\right)-a_{g_{0}}(y)\right\|<\varepsilon, \quad y \in t_{g_{1}}^{-1}(V)
$$

(one should choose $V$ in (2.14) to be in a sufficiently small neighborhood $U$ of the point $x$ and note that $\mu(V)>0$ since $\operatorname{supp} \mu=X)$.

Let $\tilde{T}_{g}$ be the operator acting in $L_{\mu}^{p}(X)$ by formula (5.1). Define $\bar{\eta} \in L_{\mu}^{p}(X, E)$ by the formula

$$
\bar{\eta}=\sum_{g \in M}\left[(\mu(V))^{-\frac{1}{p}} \tilde{T}_{g^{-1}}\left(\chi_{V}\right) \eta_{g}\right]=\sum_{g \in M} \bar{\eta}_{g},
$$

where $\chi_{V}$ is the indicator function of the set $V$. The explicit form of $\eta$ combined with (5.10), (5.9), and (5.7), show that

$$
\left\|\bar{\eta}_{g}\right\|=\left\|\eta_{g}\right\| \quad\|\bar{\eta}\|=\|\eta\|=1 .
$$

Recalling (5.3), (5.4), and (5.9), we obtain

$$
\left(b_{x} \eta\right)_{g}=0, \quad g \notin K_{1},
$$

and for each $g^{\prime} \in K_{1}$ formulas (5.3) and (5.4) yield

$$
\left(b_{x} \eta\right)_{g^{\prime}}=\sum_{g \in K} a_{g}\left(t_{g^{\prime}}^{-1}(x)\right) \eta_{g^{\prime} \cdot g} .
$$

Taking the vector $\bar{\eta}$ we have

$$
(b \bar{\eta})(y)=0 \quad \text { for each } \quad y \notin \bigcup_{g \in K_{1}} t_{g}^{-1}(V) ;
$$


moreover, for each $g^{\prime} \in K_{1}$ and $y \in t_{g^{\prime}}^{-1}(V)$ the explicit form of $\bar{\eta}$ (5.12) and of $T_{g}$ (5.1) yields

$$
\begin{aligned}
(b \bar{\eta})(y) & =\sum_{g \in K} a_{g}(y) T_{g} \bar{\eta}_{g^{\prime} \cdot g}=[\mu(V)]^{-\frac{1}{p}} \sum_{g \in K} a_{g}(y) T_{g}\left(\tilde{T}_{\left(g^{\prime} g\right)^{-1}}\left(\chi_{V}\right) \eta_{g^{\prime} g}\right) \\
& =[\mu(V)]^{-\frac{1}{p}} \sum_{g \in K} a_{g}(y) \tilde{T}_{g^{\prime-1}}\left(\chi_{V}\right) \eta_{g^{\prime} g}
\end{aligned}
$$

so that

$$
\left\|\chi_{t_{g^{\prime}}^{-1}(V)} \cdot b \bar{\eta}\right\|^{p}=\int_{t_{g^{\prime}}^{-1}(V)}\left\|\sum_{g \in K} a_{g}(y) \eta_{g^{\prime} g}\right\|^{p} \frac{\left[\tilde{T}_{g^{\prime}-1}\left(\chi_{V}\right)\right]^{p}}{\mu(V)} d \mu .
$$

Recalling that $\tilde{T}_{g^{\prime-1}}$ is an isometry in $L_{\mu}^{p}(X)$, so that

$$
\int_{t_{g^{\prime}}^{-1}(V)} \frac{\left[\tilde{T}_{g^{\prime}-1}\left(\chi_{V}\right)\right]^{p}}{\mu(V)} d \mu=1
$$

we conclude (by (5.11)) that (5.15), (5.17), and (5.18) imply that for a sufficiently small $\varepsilon$ in (5.11) and an arbitrary $\delta$,

$$
\left|\left\|\left(b_{x} \eta\right)_{g^{\prime}}\right\|^{p}-\left\|\chi_{t_{g^{\prime}}^{-1}(V)} b \bar{\eta}\right\|^{p}\right|<\delta \quad \text { for each } \quad g^{\prime} \in K_{1}
$$

Together with (5.14) and (5.16), this implies

$$
\left|\left\|b_{x} \eta\right\|^{p}-\|b \bar{\eta}\|^{p}\right|<\delta\left|K_{1}\right|
$$

and (since $\varepsilon$ and $\delta$ are arbitrary and (5.13) and (5.8) hold) the verification of inequality (5.6) is completed.

Before passing to the next step in the consideration of this example, we recall an important notion of amenability of a group.

5.3. Amenable groups. Let $G$ be a group. A state $m$ on $l^{\infty}(G)$ (i.e., a positive linear functional with norm 1) is called a left-invariant mean if

$$
m\left(f\left(s^{-1} g\right)\right)=m(f(g))
$$

for each $f \in l^{\infty}(G), s \in G$.

A group $G$ is called amenable if there exists a left-invariant mean on $l^{\infty}(G)$.

In the above definition we did not assume the existence of a topological structure on the group, i.e., $G$ was regarded as a discrete group. A general definition of amenability for locally compact topological groups, as well as further information, can be found, e.g., in 5 .

The definition of amenability presented here uses a left-invariant mean. Therefore, it is natural to view the corresponding groups as left-amenable. Using right-invariant means (i.e., functionals satisfying the condition $m(f(g s))=m(f(g)))$, we come to the notion of right-amenable groups.

In fact, these two notions coincide, i.e. (see [5, Lemma 1.1.1]),

The existence of a left-invariant mean on $l^{\infty}(G)$ is equivalent to the existence of a right-invariant mean.

There are many amenable groups. For example,

- each abelian group is amenable;

- each finite group is amenable;

- each subgroup of an amenable group is amenable;

- if $H$ is a normal subgroup of $G$ such that $H$ and $G / H$ are amenable, then $G$ is also amenable (in particular, each solvable group is amenable). 
5.4. Følner condition. There are a number of criteria of amenability of a group $G$. We will use the following Følner condition (see [5, Theorem 3.6.1]):

A group $G$ is amenable if and only if for each finite set $K \subset G$ and each $\varepsilon>0$ there exists a nonempty finite set $U$ such that

$$
\frac{|(U \cdot s) \triangle U|}{|U|}<\varepsilon \quad \text { for each } s \in K,
$$

where $|U|$ is the cardinality of $U$.

Now the next step in considering our example is the following

5.5. Lemma. Let $B\left(A, T_{g}\right)$ be the algebra described in 5.1 and $B\left(\bar{A}, V_{g}\right)$ the corresponding regular representation for $A$ and $\left\{\hat{T}_{g}\right\}_{g \in G}$ in the space $H=l^{p}\left(G, L_{\mu}^{p}(X, E)\right.$ ) (see [3.6). If $G$ is amenable, then the mapping

$$
B\left(\bar{A}, V_{g}\right) \rightarrow B\left(A, T_{g}\right)
$$

generated by the mappings

$$
\bar{a} \rightarrow a, \quad a \in A, \quad V_{g} \rightarrow T_{g}, \quad g \in G
$$

does not increase the norm.

Proof. Consider the operators

$$
b=\sum_{g \in K} a_{g} T_{g}, \quad|K|<\infty
$$

and

$$
\bar{b}=\sum_{g \in K} \bar{a}_{g} V_{g} .
$$

We need to prove that

$$
\|\bar{b}\| \geq\|b\| .
$$

Take an arbitrary $\varepsilon>0$, and let $f \in L_{\mu}^{p}(X, E)$ be a vector such that

$$
\|f\|=1
$$

and

$$
\|b f\|>\|b\|-\varepsilon .
$$

Choose an arbitrary finite set $M \subset G$ and define the vector $\eta^{M} \in H$ as follows:

$$
\eta^{M}(g)= \begin{cases}T_{g} f, & g \in M, \\ 0, & g \notin M .\end{cases}
$$

Let us note that if

$$
\left[\bigcup_{g \in K} h \cdot g\right] \subset M, \quad h \in G,
$$

then

$$
\begin{aligned}
\left\|\left(\sum_{g \in K} \bar{a}_{g} V_{g} \eta^{M}\right)(h)\right\| & =\left\|\sum_{g \in K} \hat{T}_{h}\left(a_{g}\right) \eta^{M}(h g)\right\| \\
& =\left\|\sum_{g \in K} T_{h} a_{g} T_{h g}^{-1} f\right\|=\left\|\sum_{g \in K} a_{g} T_{g} f\right\| .
\end{aligned}
$$

Recalling the Følner condition (see [5.4), let us choose finite sets $M_{n}, n=1,2, \ldots$, satisfying the condition

$$
\frac{\left|\left(M_{n} \cdot g\right) \triangle M_{n}\right|}{\left|M_{n}\right|} \underset{n \rightarrow \infty}{\longrightarrow} 0, \quad g \in K
$$


which implies

$$
\frac{\left|\bigcap_{g \in K} M_{n} \cdot g\right|}{\left|M_{n}\right|} \underset{n \rightarrow \infty}{\longrightarrow} 1,
$$

and consider the vectors $\eta^{M_{n}}$ given by (15.22).

Property (5.24), together with (5.23), means that

$$
\frac{\left\|\bar{b}\left(\eta^{M_{n}}\right)\right\|}{\left\|\eta^{M_{n}}\right\|} \underset{n \rightarrow \infty}{\longrightarrow}\|b f\| \text {. }
$$

Using (5.21) and recalling that $\varepsilon$ was arbitrary, we obtain (5.19).

The next lemma rounds up the study of our example.

5.6. Lemma. For an arbitrary element

$$
\bar{b}=\sum_{g \in K} \bar{a}_{g} V_{g} \in B\left(\bar{A}, V_{g}\right), \quad|K|<\infty,
$$

we have

$$
\|\bar{b}\|=\sup _{X}\left\|b_{x}\right\|,
$$

where $b_{x}, x \in X$, are defined in (5.2)-(5.4).

Proof. Choose an arbitrary $\varepsilon>0$, and let $\eta=\left\{\eta_{g}\right\}_{g \in G} \in H$ be a vector such that

$$
\|\eta\|=1
$$

and

$$
\|\bar{b} \eta\|>\|\bar{b}\|-\varepsilon .
$$

Without losing generality, we can assume that there exists a finite set $M \subset G$ such that

$$
\eta_{g}=0, \quad g \notin M .
$$

Recalling the natural isomorphism

$$
l^{p}\left(G, L_{\mu}^{p}(X, E)\right) \cong L_{\mu \otimes \mu^{\prime}}^{p}(X \times G, E) \cong L_{\mu}^{p}\left(X, l^{p}(G, E)\right)
$$

(where $\mu^{\prime}$ denotes the discrete measure on $G$ ), we can identify the vector $\eta=\left\{\eta_{g}\right\} \in$ $l^{p}\left(G, L_{\mu}^{p}(X, E)\right), \eta_{g} \in L_{\mu}^{p}(X, E)$, with the vector-valued function $\{\eta(x)\}_{x \in X} \in L_{\mu}^{p}(X$, $\left.l^{p}(G, E)\right)$. Using this identification, we have

$$
(\bar{b} \eta)(x)=b_{x} \eta(x)
$$

so that

$$
\|\bar{b} \eta\| \leq \sup _{X}\left\|b_{x}\right\| \cdot\|\eta\|=\sup _{X}\left\|b_{x}\right\|
$$

and by (5.28) and the arbitrariness of $\varepsilon$ we obtain the inequality

$$
\|\bar{b}\| \leq \sup _{X}\left\|b_{x}\right\| .
$$

The opposite inequality can be proved with the help of obvious modifications of arguments used in the proof of Lemma [5.2. Namely, the algebra $\bar{A}$ can be viewed as a subalgebra of the algebra $\mathrm{A}=C(X \times G, L(E))$ and the action of $G$ on $X \times G$ induced by automorphisms $V_{g}(\cdot) V_{g}^{-1}$,

$$
\bar{t}_{g}(x, \tau)=\left(x, \tau g^{-1}\right), \quad(x, \tau) \in X \times G
$$

is, clearly, topologically free.

Lemma [5.6] is proved. 
We can summarize the obtained results as the following theorem.

5.7. Theorem. Let $B\left(A, T_{g}\right)$ be the algebra defined in 5.1 and $B\left(\bar{A}, V_{g}\right)$ the corresponding regular representation of $A$ and $\left\{\hat{T}_{g}\right\}_{g \in G}$ in the space $H=l^{p}\left(G, L_{\mu}^{p}(X, E)\right)$.

If the group $G$ is amenable and the action is topologically free, then

$$
B\left(A, T_{g}\right) \cong B\left(\bar{A}, V_{g}\right)
$$

where the isomorphism is given by the mapping

$$
b=\sum_{g \in K} a_{g} T_{g} \longleftrightarrow \sum_{g \in K} \bar{a}_{g} V_{g}=\bar{b}, \quad|K|<\infty ;
$$

in particular, the algebra $B\left(A, T_{g}\right)$ possesses Properties $(*),(* *)$, and (3.1).

Proof. Apply Lemmas 5.2 5.6 and the results in Example 3.6.

\section{Example 3. Operators in $L_{\mu}^{\infty}(X, E)$}

6.1. Let $X$ be a completely regular space and $\mu$ a Borel measure on $X$ such that its support coincides with the entire $X$. Consider the Banach space $D=L_{\mu}^{\infty}(X, E)$, where $E$ is a Banach space.

Let $A=C(X, L(E))$ be the algebra of multiplication operators defined by formula (4.1) and $\left\{t_{g}\right\}_{g \in G}$ a group of homeomorphisms of $X$ preserving the equivalence class of the measure $\mu$. By $T_{g}$ we denote the isometry of the space $D$ given by the formula

$$
\left(T_{g} f\right)(x)=f\left(t_{g}^{-1}(x)\right) .
$$

Let $B\left(A, T_{g}\right) \subset L(D)$ be the Banach algebra generated by $A$ and $\left\{T_{g}\right\}_{g \in G}$. We prove that this algebra has the same properties as the algebra in Example 1.

6.2. Theorem. Let $B\left(A, T_{g}\right)$ be the algebra just introduced. If $G$ acts topologically freely, then the norm $\left\|\sum_{g \in K} a_{g} T_{g}\right\|(|K|<\infty)$ can be computed by formula (4.5).

Proof. Since $C(X, E) \subset L_{\mu}^{\infty}(X, E)$, we have (by Theorem 4.2)

$$
\left\|\sum_{g \in K} a_{g} T_{g}\right\|_{L_{\mu}^{\infty}(X, E)} \geq \sup _{X} \sup _{\left\{f_{g}\right\}_{g \in K} \in B_{K}(E)}\left\|\sum_{g \in K} a_{g}(x) f_{g}\right\| .
$$

To obtain the opposite inequality, let us note that for each $f \in L_{\mu}^{\infty}(X, E)$ with $\|f\|=1$ (without loss of generality we can also assume that $\|f\| \leq 1$ for each $x \in X$ ) we have

$$
\begin{aligned}
\left\|\sum_{g \in K} a_{g} T_{g} f\right\| & =\operatorname{essup}_{X}\left\|\sum_{g \in K} a_{g}(x) f\left(t_{g}^{-1}(x)\right)\right\| \\
& \leq \sup _{X}\left\|\sum_{g \in K} a_{g}(x) f\left(t_{g}^{-1}(x)\right)\right\| \leq \sup _{X} \sup _{\left\{f_{g}\right\}_{g \in K} \in B_{K}(E)}\left\|\sum_{g \in K} a_{g}(x) f_{g}\right\| .
\end{aligned}
$$

The proof is completed.

6.3. Remark (cf. Remark $2.3(1)$ ). If $E=\mathbf{C}$ and $G$ acts topologically freely, then

$$
\left\|\sum_{g \in K} a_{g} T_{g}\right\|=\sup _{X} \sum_{g \in K}\left|a_{g}(x)\right| .
$$

An analogue of Lemma 4.4 and Corollary 4.5 is the following result. 
6.4. Lemma. Let $B\left(A, T_{g}\right)$ be the algebra described in 6.1, and let $B\left(\bar{A}, V_{g}\right)$ be the corresponding regular representation in the space $H=l_{0}\left(G, L_{\mu}^{\infty}(X, E)\right.$ ) (or in the space $\left.l^{\infty}\left(G, L^{\infty} \mu(X, E)\right)\right)$.

If the action of $G$ is topologically free, then

$$
B\left(A, T_{g}\right) \cong B\left(\bar{A}, V_{g}\right)
$$

where the isomorphism is generated by the mappings

$$
a \rightarrow \bar{a}, \quad a \in A, \quad T_{g} \rightarrow V_{g}, \quad g \in G
$$

in particular, $B\left(A, T_{g}\right)$ possesses Properties $(*),(* *)$, and (3.1).

Proof. Cf. Lemma 4.4 and Corollary 4.5

\section{Example 4. Operators in $L_{\mu}^{1}(X, E)$}

Concluding the list of examples in the first part of the paper, we consider operators in the space $L^{1}$. In fact, this case can be reduced to the already considered case of the space $L^{\infty}$.

7.1. Let $X$ be the space with measure $\mu$ discussed in 5.1 $D=L_{\mu}^{1}(X, E), A=C(X, L(E))$ the algebra of operators defined in (4.1), and $\left\{T_{g}\right\}_{g \in G}$ operators of the form (5.1) with $p=1$.

Let $B\left(A, T_{g}\right) \subset L(D)$ be the algebra generated by $A$ and $\left\{T_{g}\right\}_{g \in G}$.

The space $L_{\mu}^{\infty}\left(X, E^{*}\right)$ (where $E^{*}$ is the space dual to $E$ ) can be isometrically embedded in $\left[L_{\mu}^{1}(X, E)\right]^{*}$, and for each $\xi \in L_{\mu}^{\infty}\left(X, E^{*}\right)$ the value of the corresponding linear functional on $f \in L_{\mu}^{1}(X, E)$ equals

$$
\langle f, \xi\rangle=\int_{X}\langle f(x), \xi(x)\rangle d \mu .
$$

Remark. We should note that in the general case

$$
\left[L_{\mu}^{1}(X, E)\right]^{*} \neq L_{\mu}^{\infty}\left(X, E^{*}\right)
$$

(see, e.g., [6, Chapter VI, Section 2,6]). The equality takes place, e.g., in the case where $\mu$ is a discrete measure or $E$ is a finite-dimensional space (other cases are presented in [6. Chapter VI, Section 2, Exercise 21]).

However, in any case, for the spaces under consideration the following equalities obviously hold:

$$
\begin{aligned}
\|f\|_{L^{1}} & =\sup _{\|\xi\|_{L^{\infty}=1}}|\langle f, \xi\rangle|, \\
\|\xi\|_{L^{\infty}} & =\sup _{\|f\|_{L^{1}}=1}|\langle f, \xi\rangle| .
\end{aligned}
$$

7.2. For an operator $a \in A$ define the operator $a^{\natural} \in L\left(L_{\mu}^{\infty}\left(X, E^{*}\right)\right)$ by the formula

$$
\left(a^{\natural} \xi\right)(x)=[a(x)]^{*} \xi(x) .
$$

Let us emphasize again that whereas $[a(x)]^{*}$ is the operator adjoint to $a(x)$, the operator $a^{\natural}$ is not necessarily adjoint to $a$ and we will call $a^{\natural}$ formally adjoint to $a$.

For each $T_{g}$ given by (5.1) (with $p=1$ ) define the operator $T_{g}^{\natural} \in L\left(L_{\mu}^{\infty}\left(X, E^{*}\right)\right.$ ) by the formula

$$
\left(T_{g}^{\natural} \xi\right)(x)=\xi\left(t_{g}(x)\right) .
$$

For the same reasons as before, we call this operator formally adjoint to $T_{g}$. 
Finally, if $b \in B\left(A, T_{g}\right)$ is of the form

$$
b=\sum_{g \in K} a_{g} T_{g}, \quad|K|<\infty,
$$

the formally adjoint to $b$ is the operator

$$
b^{\natural}=\sum_{K} T_{g}^{\natural} a^{\natural}=\sum_{K}\left[\hat{T}_{g}^{-1} a\right]^{\natural} T_{g}^{\natural},
$$

where

$$
\left(\left[\hat{T}_{g}^{-1} a\right]^{\natural} \xi\right)(x)=\left[a\left(t_{g}(x)\right)\right]^{*} \xi(x) .
$$

Let us make the following useful observation. For each $f \in L_{\mu}^{1}(X, E)$ and $\xi \in L_{\mu}^{\infty}\left(X, E^{*}\right)$ we have

$$
\langle b f, \xi\rangle=\left\langle f, b^{\natural} \xi\right\rangle,
$$

as it follows directly from the definition of $b^{\natural}$. In turn, (7.7) implies (because of (7.1) and (7.2)

$$
\|b\|_{L^{1}}=\sup _{\|f\|_{L^{1}}=1} \sup _{\|\xi\|_{L^{\infty}=1}}|\langle b f, \xi\rangle|=\sup _{\|f\|_{L^{1}}=1} \sup _{\|\xi\|_{L^{\infty}=1}}\left|\left\langle f, b^{\natural} \xi\right\rangle\right|=\left\|b^{\natural}\right\|_{L^{\infty}} .
$$

7.3. Now let $\left\{t_{g}\right\}_{g \in G}$ act topologically freely on $X$. Explicit formula (7.4) for the formally adjoint operator $T_{g}^{\natural}$ shows that the operators $T_{g}^{\natural}$ induce automorphisms that act topologically freely on $A^{\natural}$ (the algebra of operators $a^{\natural}$ formally adjoint to operators $a \in A$ ). Therefore, (77.8), (7.5), (77.6), and Theorem 6.2 imply the following result.

Let $B\left(A, T_{g}\right)$ be the algebra of operators introduced in 7.1. If the action of $G$ is topologically free, then

$$
\begin{aligned}
\left\|\sum_{g \in K} a_{g} T_{g}\right\| & =\sup _{X} \sup _{\left\{f_{g}\right\}_{g \in K} \in S_{K}\left(E^{*}\right)}\left\|\sum_{g \in K}\left[a_{g}\left(t_{g}(x)\right)\right]^{*} f_{g}\right\| \\
& =\sup _{X} \sup _{\left\{f_{g}\right\}_{g \in K} \in B_{K}\left(E^{*}\right)}\left\|\sum_{g \in K}\left[a_{g}\left(t_{g}(x)\right)\right]^{*} f_{g}\right\| .
\end{aligned}
$$

7.4. Remark (cf. Remark 6.3). If $E=\mathbf{C}$ and $G$ acts topologically freely, then

$$
\left\|\sum_{g \in K} a_{g} T_{g}\right\|=\sup _{X} \sum_{g \in K}\left|a_{g}\left(t_{g}(x)\right)\right| \text {. }
$$

Now an analogue of Lemma 6.4 is the following result.

7.5. Lemma. Let $B\left(A, T_{g}\right)$ be the algebra described in 7.1 and $B\left(\bar{A}, V_{g}\right)$ the corresponding regular representation in the space $H=l^{1}\left(G, L_{\mu}^{1}(X, E)\right)$. If $G$ acts topologically freely, then

$$
B\left(A, T_{g}\right) \cong B\left(\bar{A}, V_{g}\right),
$$

where the isomorphism is given by the mappings

$$
a \rightarrow \bar{a}, \quad a \in A, \quad T_{g} \rightarrow V_{g}, \quad g \in G
$$

in particular, $B\left(A, T_{g}\right)$ possesses Properties $(*),(* *)$, and (3.1).

Proof. The idea is the same as in the proof of Lemma 4.4 and Corollary 4.5, with the only difference that we use (7.9) instead of (4.5). 


\section{Metrichlly Free aCtion And topologically fREe ACTiON}

This and the subsequent sections 9,11 can be viewed as a "measurable analogue" of preceding sections. We continue the study of algebras of the type $B\left(A, T_{g}\right)$ in the case where $A$ is an algebra of measurable operator-valued functions on Lebesgue spaces (as opposed to objects in Section 2 and Examples 1-4, where the role of $A$ was played by algebras of continuous operator-valued functions).

In this section we show that in the "measurable" case a natural replacement of a topologically free action is the metrically free action (see 8.2). We study relations between these two notions and establish, in particular, that they are (in a sense) equivalent. This allows us to transfer main results obtained in Sections 27 from the "topological" to the "measurable" case.

8.1. Let $(\Omega, \mu)$ be a space with a $\sigma$-additive $\sigma$-finite measure $\mu, H$ a Banach space, and $L_{\mu}^{p}(\Omega, H), 1 \leq p \leq \infty$, the spaces of (equivalence classes of) measurable functions $f: \Omega \rightarrow H$ with the finite norm

$$
\|f\|=\left[\int_{\Omega}|f(x)|^{p} d \mu(x)\right]^{\frac{1}{p}}, \quad 1 \leq p<\infty, \quad\|f\|=\underset{\Omega}{\operatorname{essup}}|f|, \quad p=\infty,
$$

where $|\cdot|$ is the norm in $H$ (for details, see, e.g., [7]).

Consider the algebra $A \subset L(D)$ isomorphic to $L_{\mu}^{\infty}(\Omega, L(E))$, where $D$ and $E$ are Banach spaces. If $\left\{T_{g}\right\}_{g \in G}$ is a group of isometries of the spaces $D$ satisfying (2.1), then the automorphisms $\hat{T}_{g}$ (defined in (2.2) ) generate the mappings $\alpha_{g}: \Sigma \rightarrow \Sigma$ (where $\Sigma$ is the set of (equivalence classes of) measurable subsets $\Omega$ ) defined as follows. Let us note that for the algebra $A$ its center is $\mathbf{Z}(A) \cong L_{\mu}^{\infty}(\Omega)$. Let $\chi_{\Delta}$ be the element of the center $\mathbf{Z}(A)$ corresponding to the indicator function $\chi_{\Delta}(\omega)$ of a set $\Delta \in \Sigma$. Since $\chi_{\Delta}{ }^{2}=\chi_{\Delta}$ we see that $\hat{T}_{g}\left(\chi_{\Delta}\right)$ is a projection operator in the center $\mathbf{Z}(A)$ (which is nonzero if and only if $\chi_{\Delta} \neq 0$ ) and

$$
\hat{T}_{g}\left(\chi_{\Delta}\right)=\chi_{\tilde{\Delta}}
$$

for some $\tilde{\Delta} \in \Sigma$. We set

$$
\alpha_{g}(\Delta)=\tilde{\Delta}
$$

8.2. We say that a group $G$ acts metrically freely on an algebra $A$ (considered in 8.1) by automorphisms $\hat{T}_{g}$ (or on $\Sigma$ by mappings $\alpha_{g}$ ) if for each finite set $\left\{g_{1}, \ldots, g_{k}\right\} \subset G$ and each $\Delta \in \Sigma$ with $\mu(\Delta)>0$ there exists a set $\Delta^{\prime} \in \Sigma$ such that

(i) $\mu\left(\Delta^{\prime}\right)>0$,

(ii) $\Delta^{\prime} \subset \Delta$,

(iii) $\mu\left(\alpha_{g_{i}}\left(\Delta^{\prime}\right) \cap \alpha_{g_{j}}\left(\Delta^{\prime}\right)\right)=0, \quad i, j \in\{1, \ldots, k\}, i \neq j$.

8.3. Remark. One should note that, in a sense, the notion of a metrically free action of $G$ "coincides" with the notion of a topologically free action introduced in 2.7 .

Indeed, the algebra $L_{\mu}^{\infty}(\Omega)$ is a commutative $C^{*}$-algebra (with the natural involution). Let $M$ be the space of maximal ideals of this algebra. Then

$$
L_{\mu}^{\infty}(\Omega) \cong C(M)
$$

where the isomorphism is given by the Gelfand transform. Under this isomorphism, the indicator function $\chi_{\Delta}(\omega) \in L_{\mu}^{\infty}(\Omega)$ goes to a function $\check{\chi}_{\Delta} \in C(M)$ that is the indicator function (since $\chi_{\Delta}{ }^{2}=\chi_{\Delta}$ ) of a certain set $\check{\Delta} \subset M$. If $\mu(\Delta)>0$ (i.e., $\chi_{\Delta} \neq 0$ ), then $\check{\chi}_{\Delta}=\chi_{\check{\Delta}} \neq 0$, so that $\check{\Delta}$ is an open nonempty set. Now let us note that 
For each open nonempty set $U \subset M$ there exists a set $\Delta \in \Sigma$ such that

$$
\mu(\Delta)>0 \text { and } \check{\Delta} \subset U .
$$

Proof. First let us note that the measure $\mu$ generates a measure $\check{\mu}$ on $M$ such that

$$
\int_{\Omega} a d \mu=\int_{M} \check{a} d \check{\mu}
$$

and

$$
\operatorname{supp} \check{\mu}=M \text {. }
$$

Indeed, let

$$
\Omega=\bigcup_{i=1}^{\infty} \Omega_{i},
$$

where $\Omega_{i} \in \Sigma, \Omega_{i} \cap \Omega_{j}=\varnothing, i \neq j$, and $\mu\left(\Omega_{i}\right)<\infty$. Then the mapping

$$
\check{a} \rightarrow \int_{\Omega_{i}} a d \mu
$$

is a linear positive functional. Therefore, there exists a regular Borel measure $\check{\mu}_{i}$ on $M$ such that

$$
\int_{M} \check{a} d \mu_{i}=\int_{\Omega_{i}} a d \mu .
$$

In particular,

$$
\mu\left(\Omega_{i}\right)=\int_{\Omega} \chi_{\Omega_{i}} d \mu=\mu_{i}(M)=\mu_{i}\left(\check{\Omega}_{i}\right)
$$

and the condition $\Omega_{i} \cap \Omega_{j}=\varnothing, i \neq j$, implies that

$$
\mu_{i}\left(\check{\Omega}_{j}\right)=0 \quad \text { and } \quad \mu_{j}\left(\check{\Omega}_{i}\right)=0 .
$$

Now we set

$$
\check{\mu}=\sum_{i} \mu_{i} .
$$

By construction, the measure $\check{\mu}$ satisfies (8.4) and since

$$
\bigcup_{i=1}^{\infty} \Omega_{i}=\Omega
$$

(8.5) holds as well.

If $U=M$, then (since $\check{\Omega}=M$ ) in (8.3) we can take $\Delta=\Omega$.

Now let $U$ be an arbitrary open nonempty subset of $M, U \neq M$. Since the space $M$ is normal, there exists a nonempty open set $U_{1}$ such that

$$
U_{1} \subset \overline{U_{1}} \subset U \text { and } \overline{U_{1}} \cap[M \backslash U]=\varnothing .
$$

By the Uryson lemma, there exist functions $\check{\varphi}_{1}, \breve{\varphi}_{2} \in C(M)$ with the following properties:

(1) $0 \leq \check{\varphi}_{i}(x) \leq 1, i=1,2$,

(2) $\check{\varphi}_{1}\left(x^{\prime}\right)=1$ for some $x^{\prime} \in U_{1}$,

(3) $\check{\varphi}_{1}\left(M \backslash U_{1}\right)=0$,

(4) $\check{\varphi}_{2}\left(\overline{U_{1}}\right)=0$,

(5) $\check{\varphi}_{2}(M \backslash U)=1$. 
Properties (2), (5), and (8.5) imply that

$$
\int_{M} \check{\varphi}_{i}(x) d \check{\mu}>0, \quad i=1,2 .
$$

From Properties (3) and (4) we have

$$
\check{\varphi}_{1} \cdot \check{\varphi}_{2}=0 .
$$

In turn, (8.6), (8.4), and Property (1) imply that

$$
\int_{\Omega} \varphi_{i} d \mu=\int_{\Delta_{i}} \varphi_{i} d \mu>0, \quad i=1,2
$$

where

$$
\Delta_{i}=\left\{\omega \in \Omega: \varphi_{i}(\omega)>0\right\} .
$$

Let us note that (8.8) implies, in particular, that

$$
\mu\left(\Delta_{i}\right)>0, \quad i=1,2 .
$$

By (8.7), we have

$$
\varphi_{1} \cdot \varphi_{2}=0
$$

which means that

$$
\mu\left(\Delta_{1} \cap \Delta_{2}\right)=0 .
$$

Therefore,

$$
\chi_{\Delta_{1}} \cdot \chi_{\Delta_{2}}=0
$$

so that

$$
\check{\chi} \Delta_{1} \cdot \check{\chi} \Delta_{2}=\chi_{\check{\Delta}_{1}} \cdot \chi_{\check{\Delta}_{2}}=0 .
$$

Let us note also that by the definition of $\Delta_{i}$ we have

$$
\chi_{\Delta_{i}} \cdot \varphi_{i}=\varphi_{i}, \quad i=1,2,
$$

so that

$$
\check{\chi}_{\Delta_{i}} \cdot \check{\varphi}_{i}=\check{\varphi}_{i}, \quad i=1,2,
$$

which, in turn (by (8.12)), implies

$$
\chi_{\check{\Delta}_{1}} \cdot \check{\varphi}_{2}=\check{\chi} \Delta_{1} \cdot \check{\chi}_{\Delta_{2}} \cdot \check{\varphi}_{2},
$$

and (8.15) means, in particular, that

$$
\check{\Delta}_{1} \cap(M \backslash U)=\varnothing,
$$

i.e.,

$$
\check{\Delta}_{1} \subset U .
$$

Now from (8.10) and (8.16) we conclude that $\Delta_{1}$ is the desired set. 
Remark. Using a small modification of the above arguments, we can prove that there exists a set $\Delta \in \Sigma$ such that

$$
\mu(\Delta)>0 \text { and } \check{\Delta}=U^{\prime},
$$

establishing, therefore, a correspondence between elements in $\Sigma$ with nonzero measure and nonempty open subsets in $M$.

Now we are ready to describe what we mean by the "coincidence" of a topologically free action with a metrically free one. Namely, we prove that

A metrically free action of automorphisms $\hat{T}_{g}$ on $L_{\mu}^{\infty}(\Omega)$ corresponds to a topologically free action of automorphisms $\check{T}_{g}$ on $C(M)$ (induced by the automorphisms $\hat{T}_{g}$ and the isomorphism $\left.L_{\mu}^{\infty}(\Omega) \cong C(M)\right)$.

Proof. I. Let $\left\{\hat{T}_{g}\right\}_{g \in G}$ act metrically freely.

Consider an arbitrary finite set $\left\{g_{1}, \ldots, g_{k}\right\} \subset G$, an arbitrary open set $U \subset M$, and the set $\Delta \in \Sigma$ defined in (8.3).

Let $\Delta^{\prime} \in \Sigma$ be the set mentioned in the definition of a metrically free action (in 8.2). Condition (ii) in 8.2 and formula

$$
\chi_{\Delta^{\prime}} \cdot \chi_{\Delta}=\chi_{\Delta^{\prime}}
$$

imply that

$$
\check{\Delta}^{\prime} \subset \check{\Delta} \subset U .
$$

Condition (i) implies that $\check{\Delta}^{\prime}$ is an open set. Condition (iii) implies that

$$
\check{\chi}_{\alpha_{g_{i}}\left(\Delta^{\prime}\right)} \cdot \check{\chi}_{\alpha_{g_{j}}\left(\Delta^{\prime}\right)}=0, \quad i, j \in\{1, \ldots, k\}, i \neq j .
$$

This formula means (recalling the definition of $\alpha_{g}$ (see (8.1), (8.2)), the relation between $\hat{T}_{g}$ and $\check{T}_{g}$, and the definition of $t_{g}$ for $\check{T}_{g}$; see (2.12) $)$ that

$$
t_{g_{i}}\left(\check{\Delta}^{\prime}\right) \cap t_{g_{j}}\left(\check{\Delta}^{\prime}\right)=\varnothing, \quad i, j \in\{1, \ldots, k\}, i \neq j .
$$

Therefore (by (8.17)), condition (2.14) of a topologically free action is satisfied.

II. Now let $\left\{\check{T}_{g}\right\}_{g \in G}$ act topologically freely on $C(M)$.

Consider an arbitrary finite set $\left\{g_{1}, \ldots, g_{k}\right\} \subset G$, an element $\Delta \in \Sigma$ with $\mu(\Delta)>0$, and the set $U=\breve{\Delta} \subset M$ (as we have already seen, $U$ is a nonempty open set).

Let $V \subset U$ be the open set mentioned in (2.14). For this set we have

$$
t_{g_{i}}(V) \cap t_{g_{j}}(V)=\varnothing, \quad i, j \in\{1, \ldots, k\}, i \neq j .
$$

Take an arbitrary $\Delta^{\prime} \in \Sigma$ such that

$$
\mu\left(\Delta^{\prime}\right)>0
$$

and

$$
\check{\Delta^{\prime}} \subset V
$$

(such a set exists according to $(8.3)$ ). Let us note that the choice of $\Delta^{\prime}$ implies

$$
\check{\chi}_{\Delta} \cdot \check{\chi}_{\Delta^{\prime}}=\chi_{U} \cdot \chi_{\check{\Delta}^{\prime}}=\check{\chi}_{\Delta^{\prime}}
$$

which means that

i.e.,

$$
\chi_{\Delta} \cdot \chi_{\Delta^{\prime}}=\chi_{\Delta^{\prime}}
$$

$$
\Delta^{\prime} \subset \Delta(\bmod \mu) .
$$

Now properties (8.20) and (8.18) together with arguments used in $\mathbf{I}$ show that

$$
\mu\left(\alpha_{g_{i}}\left(\Delta^{\prime}\right) \cap \alpha_{g_{j}}\left(\Delta^{\prime}\right)\right)=0, \quad i, j \in\{1, \ldots, k\}, i \neq j,
$$


which, together with (8.19) and (8.21), proves metric freeness of the action $\left\{\hat{T}_{g}\right\}$.

Therefore the "coincidence" of topological and metric freeness is established.

Remark. It is also useful to note that a number of interesting relations between the algebras $L_{\mu}^{\infty}(\Omega)$ and $C(M)$ is described, e.g., in [8, 11.13, (f)].

An analogue of Theorem 2.8 in the metric case is the following result.

8.4. Theorem. Let an algebra $A$ and operators $T_{g}, g \in G$ be as in 8.1. If the action of $G$ is metrically free, then $B\left(A, T_{g}\right)$ possesses Property $(*)$.

Proof. It suffices to follow the proof of Theorem 2.8 using, instead of the function $c(x)$ in the proof of Theorem 2.8 appropriate sets $\Delta, \Delta^{\prime}$ and the projection $\chi_{\Delta^{\prime}}$.

\section{Example 5. Operators in $L^{p}(\Omega, E), 1<p<\infty$}

9.1. Let $(\Omega, \mu)$ be a space with a $\sigma$-additive $\sigma$-finite measure $\mu$. Consider the space $D=$ $L_{\mu}^{p}(\Omega, E), 1<p<\infty$. Let $A=L_{\mu}^{\infty}(\Omega, L(E)) \subset L(D)$ be the algebra of multiplication operators acting by the formula

$$
(a f)(x)=a(x) f(x), \quad a \in A, f \in D,
$$

and let $\alpha_{g}: \Omega \rightarrow \Omega, g \in G$, be a group of measurable mappings preserving the equivalence class of the measure $\mu$. By $T_{g}$ we denote the isometry in $D$ of the form

$$
\left(T_{g} f\right)(x)=\left[\frac{d \alpha_{g}^{-1}(\mu)}{d \mu}\right]^{\frac{1}{p}} f\left(\alpha_{g}^{-1}(x)\right),
$$

where $\frac{d \alpha_{g}^{-1}(\mu)}{d \mu}$ is the Radon-Nikodým derivative of the measure $\alpha_{g}^{-1}(\mu)$ with respect to the measure $\mu$.

One can easily see that the isometries $T_{g}$ satisfy (2.1) and the mappings $\alpha_{g}$ coincide with the mappings described in 8.1 .

Let $B\left(A, T_{g}\right) \subset L(D)$ be the Banach algebra generated by $A$ and $\left\{T_{g}\right\}_{g \in G}$.

The idea underlying the study of the present example is similar to the idea used in Example 2. Therefore, we will divide the exposition into several steps.

The first step is the following

9.2. Lemma. Let $B\left(A, T_{g}\right)$ be the algebra described in 9.1 and $B\left(\bar{A}, V_{g}\right)$ the corresponding regular representation in the space $H=l^{p}\left(G, L_{\mu}^{p}(\Omega, E)\right.$ ) (see 3.6). If the action of $G$ is metrically free, then the mapping

$$
B\left(A, T_{g}\right) \rightarrow B\left(\bar{A}, V_{g}\right)
$$

generated by the mappings

$$
a \rightarrow \bar{a}, \quad a \in A, \quad T_{g} \rightarrow V_{g}, \quad g \in G
$$

does not increase the norm.

Proof. The proof is, in a sense, an inversion of arguments used in the proof of Lemmas 5.2 and 5.6

Consider an arbitrary element $\bar{b} \in B\left(\bar{A}, V_{g}\right)$ of the form

$$
\bar{b}=\sum_{g \in K} \bar{a}_{g} V_{g}, \quad|K|<\infty .
$$

Choose $\varepsilon>0$ and let $\eta=\left\{\eta_{g}\right\} \in H$ be a vector satisfying the conditions

$$
\|\eta\|=1
$$


and

$$
\|\bar{b} \eta\|>\|\bar{b}\|-\varepsilon .
$$

Without loss of generality, we can assume that there exists a finite set $M \subset G$ such that

$$
\eta_{g}=0, \quad \text { whenever } \quad g \notin M .
$$

Recalling the arguments used in the proof of Lemma [5.6. consider the vectors $\eta$ and $\bar{b} \eta$ in the space $L_{\mu}^{p}\left(\Omega, l^{p}(G, E)\right)$.

Define a measure $\mu_{\eta}$ by the formula

$$
\mu_{\eta}(\Delta)=\int_{\Delta}\|\eta(x)\|^{p} d \mu
$$

for each measurable set $\Delta \subset \Omega$. According to (9.4) we have

$$
\mu_{\eta}(\Omega)=1 \text {. }
$$

Now (9.8) and (9.5) imply that

$$
\sup _{\eta(x) \neq 0} \frac{\|(\bar{b} \eta)(x)\|^{p}}{\|\eta(x)\|^{p}}>(\|\bar{b}\|-\varepsilon)^{p}
$$

(note that, by construction, $\bar{b}$ is a multiplication operator in $L_{\mu}^{p}\left(\Omega, l^{p}(G, E)\right.$ ), i.e., an operator acting on $\eta$ by formula (9.1)). But (9.9) means that there exists a $\mu_{\eta}$-measurable (hence also $\mu$-measurable) set $\Delta$ such that $\mu_{\eta}(\Delta)>0$ (hence also $\mu(\Delta)>0$ ) and

$$
\frac{\|(\bar{b} \eta)(x)\|}{\|\eta(x)\|}>\|\bar{b}\|-\varepsilon, \quad x \in \Delta .
$$

Let

$$
K_{1}=\bigcup_{g \in K} M \cdot g^{-1}
$$

Since the action of $G$ is metrically free, there exists a measurable set $\Delta^{\prime} \subset \Delta$ with $\mu\left(\Delta^{\prime}\right)>0$ such that

$$
\mu\left(\alpha_{g_{1}}^{-1}\left(\Delta^{\prime}\right) \cap \alpha_{g_{2}}^{-1}\left(\Delta^{\prime}\right)\right)=0,
$$

for $g_{1} \neq g_{2}$ and $g_{1}, g_{2} \in\left[K_{1} \cup M\right]$.

Consider the vector $\nu=\left\{\nu_{g}\right\} \in H$ given by the formula

$$
\nu_{g}=\chi_{\Delta^{\prime}} \cdot \eta_{g} .
$$

Since $\Delta^{\prime} \subset \Delta,(9.10)$ implies

$$
\|\bar{b} \nu\| \geq(\|\bar{b}\|-\varepsilon)\|\nu\| .
$$

Now define the vector $\bar{\nu} \in L_{\mu}^{p}(\Omega, E)$ by the formula

$$
\bar{\nu}=\sum_{g \in M}\left(T_{g^{-1}} \nu_{g}\right)=\sum_{g \in M} \bar{\nu}_{g} .
$$

By (9.11), we have

$$
\|\bar{\nu}\|=\|\nu\| .
$$

For an operator $b \in B\left(A, T_{g}\right)$ of the form

$$
b=\sum_{g \in K} a_{g} T_{g},
$$

the constriction of $\bar{\nu}$ (9.13) implies that

$$
(b \bar{\nu})(x)=0, \quad x \notin \bigcup_{g \in K_{1}} \alpha_{g}^{-1}\left(\Delta^{\prime}\right),
$$


and for $g^{\prime} \in K_{1}$ we obtain

$$
\begin{aligned}
\left\|\chi_{\alpha_{g^{\prime}}^{-1}\left(\Delta^{\prime}\right)} b \bar{\nu}\right\| & =\left\|\sum_{g \in K} a_{g} T_{g} \bar{\nu}_{g^{\prime} \cdot g}\right\|=\left\|T_{g^{\prime}}\left(\sum_{g \in K} a_{g} T_{g} T_{\left(g^{\prime} \cdot g\right)^{-1}} \nu_{g^{\prime} \cdot g}\right)\right\| \\
& =\left\|\sum_{g \in K} \hat{T}_{g^{\prime}}\left(a_{g}\right) \nu_{g^{\prime} \cdot g}\right\|=\left\|\left(\left[\sum_{g \in K} \bar{a}_{g} V_{g}\right] \nu\right)_{g^{\prime}}\right\|=\left\|(\bar{b} \nu)_{g^{\prime}}\right\|,
\end{aligned}
$$

which, together with (9.16) and (9.11), implies

$$
\|b \bar{\nu}\|=\|\bar{b} \nu\| \text {. }
$$

Recalling (9.12) and (9.14) we conclude that

$$
\|b \bar{\nu}\| \geq(\|\bar{b}\|-\varepsilon)\|\bar{\nu}\| .
$$

Since $\varepsilon$ is arbitrary, the proof is completed.

An analogue of Lemma 5.5 in our situation is the following result.

9.3. Lemma. Let $B\left(A, T_{g}\right)$ be the algebra described in 9.1 and $B\left(\bar{A}, V_{g}\right)$ the corresponding regular representation in the space $H=l^{p}\left(G, L_{\mu}^{p}(\Omega, E)\right)$. If the group $G$ is amenable, then the mapping

$$
B\left(\bar{A}, V_{g}\right) \rightarrow B\left(A, T_{g}\right)
$$

generated by the mappings

$$
\begin{gathered}
\bar{a} \rightarrow a, \quad a \in A, \\
V_{g} \rightarrow T_{g}, \quad g \in G
\end{gathered}
$$

does not increase the norm.

Proof. Similar to the proof of Lemma 5.5

We summarize the obtained results in the following theorem.

9.4. Theorem. Let $B\left(A, T_{g}\right)$ and $B\left(\bar{A}, V_{g}\right)$ be the algebras defined in Lemma 9.3. If the group $G$ is amenable and acts metrically freely, then

$$
B\left(A, T_{g}\right) \cong B\left(\bar{A}, V_{g}\right)
$$

where the isomorphism is given by mappings (9.17) and (9.18).

In particular, the algebra $B\left(A, T_{g}\right)$ possesses Properties $(*),(* *)$, and (3.1).

Proof. It suffices to apply Lemmas 9.2 and 9.3 and the results of 3.6 .

Let us recall that the study of Example 2 included the relation between the operators $b \in B\left(A, T_{g}\right), \bar{b} \in B\left(\bar{A}, V_{g}\right)$ and their representations $b_{x}, x \in X$ (see Lemmas 5.2 and [5.6), where the latter representations are defined as follows: for a point $x \in \Omega$ we define

$$
\pi_{x}: B\left(A, T_{g}\right) \rightarrow L\left(l^{p}(G, E)\right), \quad \pi_{x}(b)=b_{x}, \quad b \in B\left(A, T_{g}\right),
$$

by the formulas

$$
\begin{aligned}
\left(\pi_{x}(a) \xi\right)_{g} & =a\left(\alpha_{g}^{-1}(x)\right) \xi_{g}, \\
\left(\pi_{x}\left(T_{g_{0}}\right) \xi\right)_{g} & =\xi_{g g_{0}},
\end{aligned}
$$

where $\xi=\left(\xi_{g}\right)_{g \in G} \in l^{p}(G, E)$ and $a \in A$.

So far, in our current example we did not use analogues of the corresponding relations. To obtain these relations, we need to impose certain separability conditions on the measure $\mu$, the group $G$, and the space $E$ (the reason is that spaces of measurable functions are quite "large" and their structure is "more complicated" than the structure 
of spaces of continuous functions (it is difficult to define what we mean by the value of a measurable function at a point)).

The following lemma provides such an analogue in the measurable case.

9.5. Lemma. Let $(\Omega, \mu)$ be a space with a $\sigma$-additive $\sigma$-finite separable measure $\mu, G$ an at most countable group, and $E$ a separable Banach space. Let the algebra $A$ and the mappings $\left\{\alpha_{g}\right\}_{g \in G}$ be as in 9.1. Then for each element

$$
\bar{b}=\sum_{g \in K} \bar{a}_{g} V_{g} \in B\left(\bar{A}, V_{g}\right), \quad|K|<\infty
$$

we have

$$
\|\bar{b}\|=\operatorname{ess} \sup \left\|b_{x}\right\|
$$

where $b_{x}, x \in \Omega$, are defined in (9.19).

Proof. The inequality

$$
\|\bar{b}\| \leq \underset{\Omega}{\operatorname{ess} \sup }\left\|b_{x}\right\|
$$

can be established by arguments quite similar to those used in the proof of Lemma 5.6. Therefore the main point of the proof is to establish the opposite inequality.

Since $(\Omega, \mu)$ is a space with a separable measure, without loss of generality we can assume that the measure is strictly separable (see [9]), i.e., there exists a sequence $\Delta_{n}$ such that $\mu\left(\Delta_{n}\right)<\infty, n=1,2, \ldots$, that generates the $\sigma$-algebra of all measurable subsets in $\Omega$ (let us recall that the separability of $(\Omega, \mu)$ is equivalent to the separability of $\left.L_{\mu}^{p}(\Omega), 1 \leq p<\infty\right)$.

By $\tilde{D}$ we denote the (countable) subset of

$$
l^{p}\left(G, L_{\mu}^{p}(\Omega, E)\right) \cong L_{\mu \otimes \mu^{\prime}}^{p}(\Omega \times G, E) \cong L_{\mu}^{p}\left(\Omega, l^{p}(G, E)\right)
$$

( $\mu^{\prime}$ is the discrete measure on $G$ ) consisting of finite sums of vectors of the form

$$
\chi\left(\Delta_{n} \times g_{m}\right) e_{k}, \quad n, m, k=1,2, \ldots,
$$

where $g_{m} \in G,\left\{e_{k}\right\}$ is a (countable) dense subset of $E$ and $\chi\left(\Delta_{n} \times g_{m}\right)$ is the indicator function of the set $\Delta_{n} \times g_{m} \subset \Omega \times G$.

From the definition of $\tilde{D}$ we see that for $x \in \Omega$ the set of the vectors

$$
\{\xi(x)\}_{\xi \in \tilde{D}} \subset l^{p}(G, E)
$$

is dense in $l^{p}(G, E)$.

Now for each $f \in L_{\mu}^{\infty}(\Omega)$ and each $\eta=\{\eta(x)\}_{x \in \Omega} \in L_{\mu}^{p}\left(\Omega, l^{p}(G, E)\right)$ we have

$$
f \cdot \eta \in L_{\mu}^{p}\left(\Omega, l^{p}(G, E)\right)
$$

and

$$
\int_{\Omega}|f(x)|^{p}\|(\bar{b} \eta)(x)\|^{p} d \mu=\|\bar{b}(f \eta)(x)\|^{p} \leq\|\bar{b}\|^{p}\|f \eta\|^{p}=\|\bar{b}\|^{p} \int_{\Omega}|f(x)|^{p}\|\eta(x)\|^{p} d \mu
$$

and since $f$ is arbitrary,

$$
\left\|b_{x} \eta(x)\right\|=\|(\bar{b} \eta)(x)\| \leq\|\bar{b}\| \cdot\|\eta(x)\|
$$

for almost all $x$.

Applying this inequality to vectors $\xi \in \tilde{D}$ we obtain

$$
\left\|b_{x} \xi(x)\right\| \leq\|\bar{b}\| \cdot\|\xi(x)\| \quad \text { almost everywhere, }
$$

and since the set $\tilde{D}$ is countable and $\{\xi(x)\}_{\xi \in \tilde{D}}$ is dense in $l^{p}(G, E)$, we have

$$
\underset{\Omega}{\operatorname{ess} \sup }\left\|b_{x}\right\| \leq\|\bar{b}\|
$$

and the proof is completed. 


\section{Example 6. Operators in $L_{\mu}^{\infty}(\Omega, E)$}

10.1. Here we consider a measurable analogue of Example 3.

Let $D=L_{\mu}^{\infty}(\Omega, E)$ (where $\Omega$ is a space with a $\sigma$-additive $\sigma$-finite measure $\mu$ ), $A=$ $L_{\mu}^{\infty}(\Omega, L(E))$ the algebra of multiplication operators (9.1), and $\alpha_{g}: \Omega \rightarrow \Omega, g \in G$, a group of measurable mappings preserving the equivalence class of the measure $\mu$. By $T_{g}$ we denote the isometry of $D$ of the form

$$
\left(T_{g} f\right)(x)=f\left(\alpha_{g}^{-1}(x)\right) .
$$

Let $B\left(A, T_{g}\right)$ be the algebra generated by $A$ and $\left\{T_{g}\right\}_{g \in G}$.

For each finite subset $K \subset G$ by $B_{K}(D)$ and $S_{K}(D)$ we denote the following sets:

$$
\begin{aligned}
& B_{K}(D)=\left\{\left\{f_{g}\right\}_{g \in K}: f_{g} \in D,\left\|f_{g}\right\| \leq 1, g \in K\right\}, \\
& S_{K}(D)=\left\{\left\{f_{g}\right\}_{g \in K}: f_{g} \in D,\left\|f_{g}\right\|=1, g \in K\right\} .
\end{aligned}
$$

We begin with a theorem that claims slightly less than Theorem6.2 (in the "measurable" case) but still is an analogue of this theorem (see Remarks $10.3(2),(3)$ ).

10.2. Theorem. Let $B\left(A, T_{g}\right)$ be the algebra introduced at the beginning of this section. If the action of $G$ is metrically free, then for each finite subset $K \subset G$ we have

$$
\left\|\sum_{g \in K} a_{g} T_{g}\right\|=\sup _{\left\{f_{g}\right\}_{g} \in K \in S_{K}(D)}\left\|\sum_{g \in K} a_{g} f_{g}\right\|=\sup _{\left\{f_{g}\right\}_{g \in K} \in B_{K}(D)}\left\|\sum_{g \in K} a_{g} f_{g}\right\|,
$$

where $B_{K}(D)$ and $S_{K}(D)$ are defined in (10.2) and (10.3) respectively.

Proof. The proof is a measurable analogue of the proof of Theorem 4.2, We start with establishing the equality

$$
\left\|\sum_{g \in K} a_{g} T_{g}\right\|=\sup _{\left\{f_{g}\right\}_{g \in K} \in B_{K}(D)}\left\|\sum_{g \in K} a_{g} f_{g}\right\| .
$$

First of all, for a function $f \in L_{\mu}^{\infty}(\Omega, E)=D$ with $\|f\|=1$ we have

$$
\left\|\sum_{K} a_{g}\left(T_{g} f\right)\right\|=\left\|\sum_{K} a_{g} f_{g}\right\| \leq \sup _{\left\{f_{g}\right\}_{g} \in K \in B_{K}(D)}\left\|\sum_{K} a_{g} f_{g}\right\|,
$$

which means that

$$
\left\|\sum_{K} a_{g} T_{g}\right\| \leq \sup _{\left\{f_{g}\right\}_{g \in K} \in B_{K}(D)}\left\|\sum_{K} a_{g} f_{g}\right\| .
$$

To establish the opposite inequality, choose an arbitrary family $\left\{f_{g}\right\} \in B_{K}(D)$, and let

$$
\left\|\sum_{K} a_{g} f_{g}\right\|=\lambda \text {. }
$$

For each $\varepsilon>0$ formula (10.6) implies the existence of a measurable set $\Delta$ with $\mu(\Delta)>0$ such that

$$
\left\|\sum_{K} a_{g}(x) f_{g}(x)\right\| \geq \lambda-\varepsilon \quad \text { for each } \quad x \in \Delta .
$$

Since the action of $G$ is metrically free, there exists a measurable set $\Delta^{\prime} \subset \Delta, \mu\left(\Delta^{\prime}\right)>0$, such that

$$
\mu\left(\Delta^{\prime} \cap \alpha_{g}^{-1}\left(\Delta^{\prime}\right)\right)=0, \quad g \in K, g \neq e .
$$

Consider the function

$$
f=\sum_{K} \chi_{\alpha_{g}^{-1}\left(\Delta^{\prime}\right)} f_{g}
$$


Since $\left\{f_{g}\right\} \in B_{K}(D)$, we have

$$
\|f\| \leq 1
$$

On the other hand, the explicit form of $f$ and formula (10.7) imply that

$$
\text { for each } x \in \Delta^{\prime} \quad \text { we have }\left\|\left(\sum_{K} a_{g} T_{g} f\right)(x)\right\| \geq \lambda-\varepsilon,
$$

which, by the definition of $\lambda$ (see (10.6)) and the arbitrariness of $\varepsilon$, means that

$$
\left\|\sum_{g \in K} a_{g} T_{g}\right\| \geq \sup _{\left\{f_{g}\right\} \in B_{K}(D)}\left\|\sum_{g \in K} a_{g} f_{g}\right\|
$$

completing the verification of (10.5).

The equivalence of (10.5) and (10.4) is established using the same arguments as in the proof of Theorem 4.2 ,

The proof is completed.

10.3. Remark. (1) If $E=\mathbf{C}$ (i.e., $D=L_{\mu}^{\infty}(\Omega)$ and $A=L_{\mu}^{\infty}(\Omega)$ ), then (10.4) implies the following result:

If the action of $G$ is metrically free, then

$$
\left\|\sum_{K} a_{g} T_{g}\right\|=\operatorname{ess} \sup _{\Omega} \sum_{K}\left|a_{g}(x)\right| .
$$

Indeed, on one hand,

$$
\sup _{\left\{f_{g}\right\} \in B_{K}(D)}\left\|\sum_{g \in K} a_{g} f_{g}\right\| \leq \underset{\Omega}{\operatorname{ess} \sup } \sum_{K}\left|a_{g}(x)\right|,
$$

and to prove the opposite inequality, it suffices to set

$$
f_{g}(x)= \begin{cases}{\left[\arg a_{g}(x)\right]^{-1}} & \text { if } a_{g}(x) \neq 0, \\ 1 & \text { if } a_{g}(x)=0\end{cases}
$$

(since $a_{g} \in L_{\mu}^{\infty}(\Omega)$, we also have $f_{g} \in L_{\mu}^{\infty}(\Omega)$ ).

(2) Formula (10.4) also shows that

If the action of $G$ is metrically free, then

$$
\left\|\sum_{K} a_{g} T_{g}\right\|=\left\|\tilde{b}_{K}\right\|
$$

where the operator

$$
\tilde{b}_{K}: D_{1} \times D_{2} \times \cdots \times D_{|K|} \rightarrow D, \quad D_{i}=D
$$

is given by the formula

$$
\tilde{b}_{K}\left(\xi_{1}, \ldots, \xi_{|K|}\right)=a_{g_{1}} \xi_{1}+\cdots+a_{g_{|K|}} \xi_{|K|}
$$

$\left(K=\left\{g_{1}, \ldots, g_{|K|}\right\}\right)$.

(3) In turn, the previous remark implies the following observation.

Since $a_{g} \in L_{\mu}^{\infty}(\Omega, L(E)), g \in K$, the structure of the operator $\tilde{b}_{K}$ implies that

$$
\tilde{b}_{K} \in L_{\mu}^{\infty}(\Omega, L(\tilde{E}, E)),
$$

where $\tilde{E}=E_{1} \times E_{2} \times \cdots \times E_{|K|}, E_{i}=E$. This means that

$$
\left\|\tilde{b}_{K}(\cdot)\right\| \in L_{\mu}^{\infty}(\Omega) \quad\left\|\tilde{b}_{K}\right\|=\underset{\Omega}{\operatorname{ess} \sup }\left\|\tilde{b}_{K}(x)\right\|,
$$

and together with the previous Remark (2) we have: 
If the action of $G$ is metrically free, then

(10.11)

$$
\left\|\sum_{K} a_{g} T_{g}\right\|=\operatorname{ess} \sup _{\Omega} \sup _{\left\{f_{g}\right\} \in B_{K}(E)}\left\|\sum_{g \in K} a_{g}(x) f_{g}\right\|=\operatorname{ess} \sup _{\Omega} \sup _{\left\{f_{g}\right\} \in S_{K}(E)}\left\|\sum_{g \in K} a_{g}(x) f_{g}\right\|,
$$

thus extending Theorem 10.2 and obtaining the measurable version of Theorem 6.2 ,

We complete the consideration of our example with a measurable version of Lemma 6.4

10.4. Lemma. Let $B\left(A, T_{g}\right)$ be the algebra introduced in 10.1 and $B\left(\bar{A}, V_{g}\right)$ the corresponding regular representation in the space

$$
H=l_{0}\left(G, L_{\mu}^{\infty}(\Omega, E)\right) \quad \text { or } \quad l^{\infty}\left(G, L_{\mu}^{\infty}(\Omega, E)\right) .
$$

If the action of $G$ is metrically free, then

$$
B\left(A, T_{g}\right) \cong B\left(\bar{A}, V_{g}\right)
$$

where the isomorphism is generated by the mappings

$$
a \rightarrow \bar{a}, \quad a \in A, \quad T_{g} \rightarrow V_{g}, \quad g \in G
$$

in particular, the algebra $B\left(A, T_{g}\right)$ possesses Properties $(*)$, (**), and (3.1).

Proof. Direct computations show that the norm of the element

$$
\bar{b}=\sum_{g \in K} \bar{a}_{g} V_{g} \in B\left(\bar{A}, V_{g}\right)
$$

equals

$$
\sup _{\left\{f_{g}\right\} \in B_{K}(D)}\left\|\sum_{g \in K} a_{g} f_{g}\right\|
$$

thus establishing an isomorphism

$$
B\left(A, T_{g}\right) \cong B\left(\bar{A}, V_{g}\right)
$$

Properties $(*),(* *)$, and (3.1) of the algebra $B\left(A, T_{g}\right)$ follow from the results in 3.6

\section{EXAmple 7. Operators in $L_{\mu}^{1}(\Omega, E)$}

Our last example deals with the space $L^{1}$ and is, in fact, a measurable version of Example 4. The arguments below are similar to those used in Example 4 (i.e., we reduce this case to the already studied case $L^{\infty}$ ). However, these arguments require using the results of the previous section instead of the results of Example 3.

11.1. Let $(\Omega, \mu)$ be the space introduced in 9.1 $D=L_{\mu}^{1}(\Omega, E), A=L_{\mu}^{\infty}(\Omega, L(E))$ the operator algebra defined in (9.1), and $T_{g}$ as in (9.2) (with $p=1$ ). Let $B\left(A, T_{g}\right) \subset L(D)$ be the algebra generated by $A$ and $\left\{T_{g}\right\}_{g \in G}$.

11.2. Recalling arguments used in Example 4 (i.e., introducing the corresponding formally adjoint operators) we can obtain the following result (which is a natural analogue of results in 7.3 for operators under consideration).

Let $B\left(A, T_{g}\right)$ be the algebra introduced in 11.1, If the action of $G$ is metrically free, then

$$
\begin{aligned}
\left\|\sum_{g \in K} a_{g} T_{g}\right\| & =\underset{\Omega}{\operatorname{ess} \sup _{\Omega}} \sup _{\left\{f_{g}\right\} \in B_{K}\left(E^{*}\right)}\left\|\sum_{g \in K}\left[a_{g}\left(\alpha_{g}(x)\right)\right]^{*} f_{g}\right\| \\
& =\operatorname{ess} \sup _{\Omega} \sup _{\left\{f_{g}\right\} \in S_{K}\left(E^{*}\right)}\left\|\sum_{g \in K}\left[a_{g}(\alpha(x))\right]^{*} f_{g}\right\| .
\end{aligned}
$$


Proof. It suffices to follow the arguments in 7.3 using Remark 10.3 (3) instead of Theorem 6.2

11.3. Remark (cf. Remark 10.3 (1)). If $E=\mathbf{C}$ and the action of $G$ is metrically free, then

$$
\left\|\sum_{K} a_{g} T_{g}\right\|=\underset{\Omega}{\operatorname{ess} \sup } \sum_{K}\left|a_{g}\left(\alpha_{g}(x)\right)\right| .
$$

Finally, an analogue of Lemma 10.4 is the following result.

11.4. Lemma. Let $B\left(A, T_{g}\right)$ be the algebra described in 11.1 and $B\left(\bar{A}, V_{g}\right)$ the corresponding regular representation in the space $l^{1}\left(G, L_{\mu}^{1}(\Omega, E)\right)$. If the action of $G$ is metrically free, then

$$
B\left(A, T_{g}\right) \cong B\left(\bar{A}, V_{g}\right)
$$

where the isomorphism is generated by the mappings

$$
a \rightarrow \bar{a}, \quad a \in A, \quad T_{g} \rightarrow V_{g}, \quad g \in G
$$

in particular, $B\left(A, T_{g}\right)$ possesses Properties $(*)$, (**), and (3.1).

\section{INTERPOLATION}

In Examples 2 and 5 we did not obtain an explicit formula for the norm of the operator $T_{g}$. However, formulas obtained in the consideration of other examples, together with the Riesz-Thorin Interpolation Theorem, allow us to obtain useful estimates for the norm of operators of the form $\sum a_{g} T_{g}$ in all cases.

To present the corresponding results, we introduce, first of all, the space $L_{\mu}^{0}(\Omega, E)$ that is the closed subspace of the space $L_{\mu}^{\infty}(\Omega, E)$ generated by the functions with support of finite measure, i.e., by functions $f$ such that

$$
f \in L_{\mu}^{\infty}(\Omega, E) \text { and } \mu(\{x: f(x) \neq 0\})<\infty .
$$

It is clear that if $\mu$ is a discrete measure, then

$$
L_{\mu}^{0}(\Omega, E)=l_{0}(\Omega, E) .
$$

The next Theorem 12.1 is, in fact, a vector-valued version of the classical Riesz-Thorin Interpolation Theorem (see [10, 1.1.1]).

12.1. Theorem. Let $V$ be a linear operator that belongs both to $L\left(D_{1}\right)$ and to $L\left(D_{2}\right)$, where $D_{i}=L_{\mu}^{p_{i}}(\Omega, E)$ and $p_{1} \in[1, \infty), p_{2} \in[1, \infty) \cup\{0\}$. For each $\Theta, 0<\Theta<1$, define the number $p$ by the formula

$$
\frac{1}{p}=\frac{1-\Theta}{p_{1}}+\frac{\Theta}{p_{2}} \quad \text { if } \quad p_{2} \neq 0
$$

or

$$
\frac{1}{p}=\frac{1-\Theta}{p_{1}} \quad \text { if } \quad p_{2}=0
$$

and let $D=L_{\mu}^{p}(\Omega, E)$.

Then the operator $V$ belongs to $L(D)$ and

$$
\|V\|_{D} \leq\|V\|_{D_{1}}^{1-\Theta} \cdot\|V\|_{D_{2}}^{\Theta} .
$$

Proof. See [10, 5.1.2 and 4.1.2]. 
Let us apply this result to the operators in Example 2.

Let $b \in B\left(A, T_{g}\right) \subset L\left(L_{\mu}^{1}(X, E)\right), 1 \leq p \leq \infty$, be the operator of the form

$$
b=\sum_{g \in K} a_{g} T_{g}, \quad|K|<\infty,
$$

where for $1 \leq p<\infty$, the algebra $B\left(A, T_{g}\right)$ is described in 5.1, and for $p=\infty$, in 6.1,

Also let $\bar{b} \in B\left(\bar{A}, V_{g}\right)$ be the operator of the form

$$
\bar{b}=\sum_{g \in K} \bar{a}_{g} V_{g},
$$

where $B\left(\bar{A}, V_{g}\right)$ is the corresponding regular representation for $A$ and $\left\{\hat{T}_{g}\right\}_{g \in G}$ in the space $l^{p}\left(G, L_{\mu}^{p}(X, E)\right), 1 \leq p \leq \infty$ (in the case $p=\infty$ we can also use the space $\left.l^{0}\left(G, L_{\mu}^{\infty}(X, E)\right)\right)$.

By $\|b\|_{p}$ we denote the norm of the operator $b$ acting in the space $L_{\mu}^{p}(X, E)$. Similarly, we introduce the notation $\|\bar{b}\|_{p}$.

An important observation is that the operator $\bar{b}$ is given by the same formula in all cases $1 \leq p \leq \infty$ (which allows us to apply Theorem 12.1).

Let us recall that results obtained in the consideration of Examples 2-4 show that if the group $G$ is amenable and the action of $G$ is topologically free, then for $p \in[1, \infty]$ we have

$$
\|b\|_{p}=\|\bar{b}\|_{p}
$$

(in the cases $p=1$ and $p=\infty$ we do not need the amenability of $G$ ). This formula, the just mentioned properties of the structure of the operator $\bar{b}$, and Theorem 12.1imply the following result.

12.2. Lemma. If the group $G$ is amenable and the action of $G$ on $X$ is topologically free, then for each $p \in(1, \infty)$ we have

$$
\|b\|_{p} \leq\|b\|_{1}^{\frac{1}{p}} \cdot\|b\|_{\infty}^{1-\frac{1}{p}}
$$

where $\|b\|_{1}$ is defined in (7.9) and $\|b\|_{\infty}$ in (4.5).

12.3. Analyzing Example 2 more closely, we can improve the estimate (12.4). Namely, let us recall that if $G$ is amenable and the action of $G$ is topologically free, results of Examples 2, 3, and 4 show also that

$$
\|b\|_{p}=\|\bar{b}\|_{p}=\sup _{X}\left\|b_{x}\right\|_{p}
$$

where $b_{x}$ is defined in (5.2)-(5.4) (we consider this operator as acting in the corresponding space $l^{p}(G, E), 1 \leq p \leq \infty$, or, in the case $p=\infty$, in the space $\left.l_{0}(G, E)\right)$.

For the operators $b_{x}$, Theorem 12.1 implies the following result:

For each $p \in(1, \infty)$ we have

$$
\left\|b_{x}\right\|_{p} \leq\left\|b_{x}\right\|_{1}^{\frac{1}{p}} \cdot\left\|b_{x}\right\|_{\infty}^{1-\frac{1}{p}}=\left\|b_{x}\right\|_{1}^{\frac{1}{p}} \cdot\left\|b_{x}\right\|_{0}^{1-\frac{1}{p}}
$$

(here $\left\|b_{x}\right\|_{0}$ is the norm of the operator $b_{x}$ in $l_{0}(G, E)$ ).

By (12.5) this means that

If the group $G$ is amenable and the action of $G$ on $X$ is topologically free, then for each $p \in(1, \infty)$ we have

$$
\|b\|_{p}=\|\bar{b}\|_{p} \leq \sup _{X}\left\|b_{x}\right\|_{1}^{\frac{1}{p}} \cdot\left\|b_{x}\right\|_{\infty}^{1-\frac{1}{p}} .
$$


Let us recall also that the results obtained in considering Examples 1 and 4 show that

$$
\begin{aligned}
\left\|b_{x}\right\|_{\infty} & =\left\|b_{x}\right\|_{0}=\sup _{h \in G} \sup _{\left\{f_{g}\right\}_{g \in K} \in S_{K}(E)}\left\|\sum_{g \in K} a_{g}\left(t_{h}^{-1}(x)\right) f_{g}\right\| \\
& =\sup _{h \in G} \sup _{\left\{f_{g}\right\}_{g \in K} \in B_{K}(E)}\left\|\sum_{g \in K} a_{g}\left(t_{h}^{-1}(x)\right) f_{g}\right\|
\end{aligned}
$$

and

$$
\begin{aligned}
\left\|b_{x}\right\|_{1} & =\sup _{h \in G} \sup _{\left\{f_{g}\right\}_{g \in K} \in S_{K}\left(E^{*}\right)}\left\|\sum_{g \in K}\left[a_{g}\left(t_{g \cdot h^{-1}}(x)\right)\right]^{*} f_{g}\right\| \\
& =\sup _{h \in G} \sup _{\left\{f_{g}\right\}_{g \in K} \in B_{K}\left(E^{*}\right)}\left\|\sum_{g \in K}\left[a_{g}\left(t_{g \cdot h^{-1}}(x)\right)\right]^{*} f_{g}\right\| .
\end{aligned}
$$

12.4. Now let $b \in B\left(A, T_{g}\right) \subset L\left(L_{\mu}^{p}(\Omega, E)\right), 1 \leq p \leq \infty$, be an operator of the form (12.2), where for $1 \leq p<\infty$, the algebra $B\left(A, T_{g}\right)$ is described in 9.1, and for $p=\infty$, in 10.1. Here by $\|b\|_{p}$ we denote the norm of $b$ as an operator in the space $L_{\mu}^{p}(\Omega, E)$ ).

A measurable analogue of Lemma 12.2 for all these objects is the following result.

12.5. Lemma. If the group $G$ is amenable and the action of $G$ on $\Omega$ is metrically free, then for each $p \in(1, \infty)$ we have

$$
\|b\|_{p} \leq\|b\|_{1}^{\frac{1}{p}} \cdot\|b\|_{\infty}^{1-\frac{1}{p}}
$$

where $\|b\|_{1}$ is defined by (11.1) and $\|b\|_{\infty}$ by (10.11).

\section{Concluding Remarks. Isomorphism theorems}

Here we present concluding remarks and discuss some relations between the examples considered in preceding sections and the isomorphism theorem [3, Corollary 12.17].

13.1. (1) Let us note that in Examples 1, 3, 4, 6, 7 we did not use any particular properties of the group $G$, so that in these examples $G$ could have been an arbitrary, not necessarily amenable, group

(2) A completely different picture shows up in Examples 2 and 5.

Here

(i) if $G$ acts topologically (metrically) freely, then $B\left(\bar{A}, V_{g}\right)$ is a representation of $B\left(A, T_{g}\right)$ for an arbitrary group $G$ (not necessarily amenable) (Lemmas 5.2, 5.6. and 9.2).

On the other hand,

(ii) if the group $G$ is amenable, then $B\left(A, T_{g}\right)$ is a representation of $B\left(\bar{A}, V_{g}\right)$ for each action of $G$ (not necessarily topologically (metrically) free) (Lemmas 5.5) and 9.3).

Therefore, in these two examples the topological (metric) freeness of the action of the group $G$ and the amenability of $G$ are, in a sense, complementary: if $G$ acts metrically freely, then $B\left(A, T_{g}\right)$ is "larger" than $B\left(\bar{A}, V_{g}\right)$ (see (i)), and if $G$ is amenable, then $B\left(\bar{A}, V_{g}\right)$ is "larger" than $B\left(A, T_{g}\right)$ (see (ii)).

These two algebras "coincide" if $G$ is amenable and acts topologically (metrically) freely (Theorems 5.7 and 9.4). 
13.2. The consideration of Examples 2 and 5 leads to the results of the Isomorphism Theorem type (as in [3, Corollary 12.17]), which establish an isomorphism between operator algebras acting in "completely different" spaces (so, in a sense, "eliminating" these spaces)

For the situation considered in Example 2, this result looks as follows.

Let $\mu_{1}$ and $\mu_{2}$ be two Borel measures on a completely regular space $X$ such that the support of each of these measures coincides with $X$. Also let $\left\{t_{g}\right\}_{g \in G}$ be a group of diffeomorphisms of $X$ preserving the class of each of the measures $\mu_{1}$ and $\mu_{2}$. Consider the space $D_{i}=L_{\mu_{i}}^{p}(X, E), i=1,2$. Let $A_{i}=C\left(X, L(E) \subset L\left(D_{i}\right)\right), i=1,2$, be the algebras of multiplication operators given by formula (4.1), $T_{g}^{i}, i=1,2$, isometries of $D_{i}$ of the form (5.1) (with $\left.\mu=\mu_{i}\right)$ and $B\left(A_{i}, T_{g}^{i}\right), i=1,2$ the algebras generated by $A_{i}$ and $\left\{T_{g}^{i}\right\}_{g \in G}$.

The isomorphism theorem we have in mind is formulated as follows.

If the group $G$ is amenable and acts topologically freely, then $B\left(A_{1}, T_{g}^{1}\right)$ and $B\left(A_{2}, T_{g}^{2}\right)$ are isomorphic (as Banach algebras) and the isomorphism is given by the natural isomorphism

$$
A_{1} \cong A_{2}
$$

and the mappings

$$
T_{g}^{1} \rightarrow T_{g}^{2} .
$$

Proof. Lemmas 5.2, 5.5, and 5.6 imply that

$$
B\left(A_{i}, T_{g}^{i}\right) \cong \bigoplus_{x \in X} \pi_{x}\left(B\left(A_{i}, T_{g}^{i}\right)\right),
$$

where $\pi_{x}$ is defined in (5.2) (5.4). Let us note that the explicit form of $\pi_{x}$ shows that the right-hand side of (13.1) depends only on $\left\{t_{g}\right\}_{g \in G}$ and not on $\mu_{i}, i=1,2$, thus proving our claim.

In the case of Example 5 the corresponding result is formulated as follows.

Let $\left(\Omega, \mu_{i}\right), i=1,2$, be two spaces with $\sigma$-additive $\sigma$-finite separable measures $\mu_{1}$ and $\mu_{2}$ that are mutually absolutely continuous (i.e., $L_{\mu_{1}}^{\infty}(\Omega, L(E)) \cong L_{\mu_{2}}^{\infty}(\Omega, L(E))$ ), and let $\left\{\alpha_{g}\right\}_{g \in G}$ be a group of measurable mappings of the space $\Omega$, each preserving the equivalence classes of the measures $\mu_{1}$ and $\mu_{2}$. Let us consider the spaces $D_{i}=L_{\mu_{i}}^{p}(\Omega, E)$, $i=1,2$. Let $A_{i}=L_{\mu_{1}}^{\infty}(\Omega, L(E)) \subset L\left(D_{i}\right)$ be the algebras of the multiplication operators given by formula (9.1) $, T_{g}^{i}, i=1,2$, isometries of $D_{i}$ of the form (9.2) (with $\mu=\mu_{i}$ ) and $B\left(A, T_{g}^{i}\right)$ the algebras generated by $A_{i}$ and $\left\{T_{g}^{i}\right\}_{g \in G}$.

The isomorphism theorem for this case is formulated as follows.

Let $E$ be a separable Banach space, $G$ an at most countable amenable group acting metrically freely on $E$. Then $B\left(A_{1}, T_{g}^{1}\right)$ and $B\left(A_{2}, T_{g}^{2}\right)$ are isomorphic (as Banach algebras) and the isomorphism is given by the natural isomorphism

$$
A_{1} \cong A_{2}
$$

and the mappings

$$
T_{g}^{1} \rightarrow T_{g}^{2} .
$$

Proof. The proof follows from Lemma 9.5 .

\section{REFERENCES}

[1] G. K. Pedersen, $C^{*}$-algebras and their automorphism groups. Academic Press, London-New York, 1979. MR0548006 (81e:46037)

[2] M. B. Landstad, Duality theory for covariant systems. Trans. Amer. Math. Soc. 248 (1979) 223-267. MR0522262 (80j:46107) 
[3] A. Antonevich and A. Lebedev, Functional differential equations: I. $C^{*}$-theory. Longman, Harlow, 1994. MR 1413495 (97e:46072)

[4] E. Hewitt and K. Ross, Abstract harmonic analysis. Vol. I. Springer-Verlag, Berlin-New York, 1979. MR0551496 (81k:43001)

[5] F. P. Greenleaf, Invariant means on topological groups and their applications. Van Nostrand, New York-Toronto-London, 1969. MR0251549(40:4776)

[6] N. Bourbaki, Intégration. Ch. I-IV. Hermann, Paris, 1952. MR0054691 (14:960h)

[7] N. Dunford and J. Schwartz, Linear operators. Part I. Wiley, New York, 1958. MR1009162 (90g:47001a)

[8] W. Rudin, Functional analysis. McGraw-Hill, New York-Düsseldorf-Johannesburg, 1973. MR0365062 (51:1315)

[9] P. R. Halmos, The decomposition of measures. Duke Math. J. 8 (1941) 386-392. MR0004718(3:50e)

[10] J. Bergh and J. Löfström, Interpolation spaces. An introduction. Springer-Verlag, Berlin-New York, 1976. MR.0608963 (82c:46083)

Belarus State University, Minsk 220050, Belarus

Current address: Institute of Mathematics, University of Białystok, Akademicka 2, Białystok 15-267, Poland

E-mail address: lebedev@bsu.by

Translated by O. KHLEBORODOVA 